\title{
Disrupted Blood-Retina Lysophosphatidylcholine Transport Impairs Photoreceptor Health But Not Visual Signal Transduction
}

\author{
Ekaterina S. Lobanova, ${ }^{1,2}$ Kai Schuhmann, ${ }^{4}$ Stella Finkelstein, ${ }^{5}$ Tylor R. Lewis, ${ }^{5}$ Martha A. Cady, ${ }^{5}$ Ying Hao, ${ }^{5}$ \\ Casey Keuthan, ${ }^{1,3} \oplus^{\oplus}$ John D. Ash, ${ }^{1,3}{ }^{\oplus}$ Marie E. Burns, ${ }^{7}$ Andrej Shevchenko, ${ }^{4}$ and $\odot$ Vadim Y. Arshavsky ${ }^{5,6}$ \\ Departments of ${ }^{1}$ Ophthalmology, ${ }^{2}$ Pharmacology and Therapeutics, ${ }^{3}$ Molecular Genetics and Microbiology, University of Florida, Gainesville, Florida, \\ 32610, ${ }^{4}$ Max Planck Institute of Molecular Cell Biology and Genetics, Dresden, Germany, 01307, Departments of ${ }^{5}$ Ophthalmology, ${ }^{6}$ Pharmacology and \\ Cancer Biology, Duke University, Durham, North Carolina, 27710, and ${ }^{7}$ Department of Cell Biology and Human Anatomy, University of California-Davis, \\ Davis, California, 95618
}

Retinal photoreceptor cells contain the highest concentration of docosahexaenoic acid(DHA) in our bodies, and it has been long assumed that this is critical for supporting normal vision. Indeed, early studies using DHA dietary restriction documented reduced light sensitivity by DHA-deprived retinas. Recently, it has been demonstrated that a major route of DHA entry in the retina is the delivery across the blood-retina barrier by the sodium-dependent lipid transporter, Mfsd2a. This discovery opened a unique opportunity to analyze photoreceptor health and function in DHA-deprived retinas using the $M f s d 2 a$ knock-out mouse as animal model. Our lipidome analyses of $M f s d 2 a^{-/-}$retinas and outer segment membranes corroborated the previously reported decrease in the fraction of DHA-containing phospholipids and a compensatory increase in phospholipids containing arachidonic acid. We also revealed an increase in the retinal content of monounsaturated fatty acids and a reduction in very long chain fatty acids. These changes could be explained by a combination of reduced DHA supply to the retina and a concomitant upregulation of several fatty acid desaturases controlled by sterol regulatory element-binding transcription factors, which are upregulated in $M f s d 2 a^{-/-}$retinas. $M f s d 2 a^{-/-}$retinas undergo slow progressive degeneration, with $\sim 30 \%$ of photoreceptor cells lost by the age of 6 months. Despite this pathology, the ultrastructure $M f s d 2 a^{-/-}$photoreceptors and their ability to produce light responses were essentially normal. These data demonstrate that, whereas maintaining the lysophosphatidylcholine route of DHA supply to the retina is essential for long-term photoreceptor survival, it is not important for supporting normal phototransduction.

Key words: DHA; Mfsd2a; phospholipids; photoreceptor; retina; retinal degeneration

\section{Significance Statement}

Phospholipids containing docosahexaenoic acid (DHA) are greatly enriched in the nervous system, with the highest concentration found in the light-sensitive membranes of photoreceptor cells. In this study, we analyzed the consequences of impaired DHA transport across the blood-retina barrier. We have found that, in addition to a predictable reduction in the DHA level, the affected retinas undergo a complex, transcriptionally-driven rebuilding of their membrane lipidome in a pattern preserving the overall saturation/desaturation balance of retinal phospholipids. Remarkably, these changes do not affect the ability of photoreceptors to produce responses to light but are detrimental for the long-term survival of these cells.

\section{Introduction}

There is ample evidence supporting the health benefits of consuming $\omega$-3 fats, i.e., phospholipids containing docosahexaenoic acid (DHA) moieties. DHA is highly concentrated in the nervous system despite the fact that DHA cannot be synthetized by neurons and has to be transported to the brain across the bloodbrain barrier (BBB). A major breakthrough in understanding this transport was reported by Nguyen et al. (2014) who discovered 
that facilitative transporter Mfsd2a, expressed in the endothelium of the BBB microvessels, is responsible for the uptake of plasma lysophosphatidylcholines (LPCs) across the BBB, thereby serving as a primary mechanism of DHA delivery to the brain. Mfsd2a knock-out, as well as its mutations, results in microcephaly and hypomyelination in animal models and human patients (Nguyen et al., 2014; Alakbarzade et al., 2015; Guemez-Gamboa et al., 2015; Chan et al., 2018; Harel et al., 2018).

Remarkably, the retina contains even more DHA than the brain, with the highest concentration found in the light-sensitive outer segment membranes of rod and cone photoreceptor cells (Fliesler and Anderson, 1983). It is generally agreed that, like the brain, the retina cannot synthesize its own DHA de novo and relies on uptake from extraretinal sources, such as blood-borne lipids, for maintaining its DHA content (Scott and Bazan, 1989; Bazan et al., 2011). Multiple studies analyzing the consequences of DHA dietary restriction documented that DHA-deprived retinas produced light responses of reduced sensitivity (Benolken et al., 1973; Wheeler et al., 1975; Senapati et al., 2018), suggesting that DHA may be critical for supporting visual signaling. Recently, Silver and colleagues showed that Mfsd2a is expressed in retinal pigment epithelium (RPE) and retinal vasculature and that the Mfsd2a-mediated lipid transport in RPE is particularly important for maintaining the high DHA concentration in the retina (Wong et al., 2016). They reported that whole eyes of $M f_{s} d 2 a^{-/-}$mice contain $\sim 40 \%$ less DHA than WT controls and that this decrease is compensated by an increase in the content of arachidonic acid (AA). They also reported that $M f_{s} d 2 a^{-/-}$photoreceptors appear to have a morphological defect in their outer segment disc structure. Yet, their analysis of visual function by electroretinography yielded nearly normal light responses.

Here, we analyzed the retinal phenotype of an alternative $M f_{s} d 2 a^{-1-}$ mouse strain, which corroborated and expanded several key observations from the previous report (Wong et al., 2016), disagreed with two and revealed a number of novel findings (for a detailed comparison between the two studies, see Discussion). We have found that $M f_{s} d 2 a^{-/-}$retinas undergo a global remodeling of their fatty acid composition, including a $45 \%$ reduction in the fraction of DHA lipids and a compensatory $66 \%$ increase in the fraction of AA. We also observed a $56 \%$ increase in the fraction of phospholipids containing monounsaturated fatty acid moieties and a 57\% loss in phospholipids containing very long chain fatty acid moieties. Similar changes were observed in an independently analyzed lipidome of isolated photoreceptor outer segment membranes. $M f s d 2 a^{-/-}$photoreceptors underwent relatively slow progressive degeneration, although surviving photoreceptors retained an entirely normal ultrastructure. Remarkably, single-cell recording from $M f s d 2 a^{-/-}$rods did not reveal any major phototransduction phenotype. This indicates that impaired LPC supply to the retina and the corresponding $\sim$ twofold reduction in the content of retinal DHA are detrimental for long-term viability of photoreceptor cells, but do not impact the ability of surviving cells to produce normal responses to light.

of Florida and Duke University; the German Research Foundation TRR83 Grant project A17 (A.S.); and Federal Ministry of Education and Research LIFS Grant (A.S.). We thank Dr. David L Silver (Duke NUS) for many helpful discussions and critical reading of the paper.

The authors declare no competing financial interests

Correspondence should be addressed to Ekaterina S. Lobanova at elobanova@ufl.edu or Vadim Y. Arshavsky at vadim.arshavsky@duke.edu.

https://doi.org/10.1523/JNEUROSCl.1142-19.2019

Copyright $\odot 2019$ the authors

\section{Materials and Methods}

Animals. Frozen embryos of $M f s d 2 a$ heterozygote mice were purchased from the KOMP repository (032467-UCD) and recovered by the Duke Transgenics Core. One of the recovered heterozygous female mice was used as a founder. This is the same line as used by Ben-Zvi et al. (2014) and Chow and $\mathrm{Gu}$ (2017), except that we backcrossed the line used in this study to the C57BL/6J background (Jackson Laboratories, stock $\# 000664)$ for eight generations. The line was negative for $r d 1$ and $r d 8$ mutations. Mice were maintained on the Teklad global 18\% protein rodent diet 2918 (Envigo). At 1 month of age $M f_{s} d 2 a^{-1-}$ mice were on average $\sim 25 \%$ smaller than their WT littermates [ $14.8 \pm 1.1 \mathrm{~g}$ vs $19.7 \pm$ $0.9 \mathrm{~g}$ for $M f_{s} d 2 a^{-/-}$vs $M f_{s} d 2 a^{+/+}$males (mean $\left.\pm \mathrm{SD} ; n=4\right) ; 12.7 \pm$ $1.3 \mathrm{~g}$ vs $17 \pm 1.2 \mathrm{~g}$ for $M f_{s} d 2 a^{-/-}$vs $M f_{s} d 2 a^{+/+}$females $\left.(n=5)\right]$. Knockout and WT littermate mice used for experiments were obtained by in-crossing the $M f_{s} d 2 a^{+/-}$mice. Note, that $M f_{s} d 2 a^{-/-}$mice in our hands could produce progeny as well. Based on monitoring three $M f_{s} d 2 a^{-1-}$ breeding pairs, we noted that one breeding pair produced no litters, whereas others produced one to two litters with one or two surviving mice. $R h o^{P 23 H / P 23 H}$ mice were purchased from Jackson Laboratories (stock \#017628). Animals for experiments were obtained by mating Rho ${ }^{P 23 H / W T}$ mice with C57BL/6J WT mice (Jackson Laboratories, stock \#000664). Mouse genotypes were determined using real time PCR with specific probes designed for each gene (Transnetyx).

Animals were reared under a normal day/night cycle and handled according to the protocols approved by the Institutional Animal Care and Use Committees of Duke University and the University of Florida.

Antibodies. For Western blotting, mouse monoclonal antibody 4D2 against rhodopsin (1:5000) was a gift from R.S. Molday (University of British Columbia). Rabbit anti-G $\alpha_{\mathrm{t}}(\mathrm{sc}-389 ; 1: 10,000)$ and antiphosducin (sc-23774; 1:5000) antibodies were from Santa Cruz Biotechnology. Rabbit anti-arrestin antibody (PA1-7; 1:5000) was from Affinity Bioreagents. Rabbit antibodies against retinal guanylate cyclase isoforms 1 (1:2000) and 2 (1:2000) were a gift from A. M. Dizhoor (Pennsylvania College of Optometry). Rabbit anti-heat shock 70 protein antibodies (ADI-SPA-816; 1:5000) were from Enzo Life Sciences. Secondary goat or donkey antibodies for Western blotting conjugated with AlexaFluor 680 (A-21057) and 800 (SA5-10044; both 1:10,000) were from Invitrogen.

For immunohistochemistry, we used mouse monoclonal antibody 1D4 against rhodopsin (ab5417; 1:1000) from Abcam. Secondary donkey antibodies conjugated with AlexaFluor 488 (A-21202; 1:1000) were from Life Technologies.

Histology and microscopy. Mice were killed by $\mathrm{CO}_{2}$ and transcardially perfused with $3 \mathrm{ml}$ of $2 \%$ paraformaldehyde and $2.5 \%$ glutaraldehyde solution in PBS at a flow rate of $1-2 \mathrm{ml} / \mathrm{min}$. The superior-inferior line was marked with a burnmark using low temperature cautery (Bovie, AA90). Next, eyes were enucleated, exterior tissue was removed, and eyes were fixed overnight at $4^{\circ} \mathrm{C}$ in $2 \%$ paraformaldehyde and $2.5 \%$ glutaraldehyde in PBS. The next day, eyes were rinsed with PBS, cornea and lens were removed, treated with $2 \%$ osmium tetroxide (Petters et al., 1997) and embedded in EPON 812 (Electron Microscopy Sciences). Plasticembedded retinal cross-sections $(0.5 \mu \mathrm{m})$ were stained with toluidine blue for light microscopy (Lobanova et al., 2013). Nuclear count was performed in sections cut through the optic nerve in $100 \mu \mathrm{m}$ segments of the outer nuclear layer at $500 \mu \mathrm{m}$ steps from the optic nerve head as described by Lobanova et al. (2018). For electron microcopy, 60-80 nm ultrathin sections were cut from the same blocks with an ultramicrotome (Leica), collected on copper grids (Electron Microscopy Sciences) and post-stained with $1 \%$ uranyl acetate and Sato's lead (Sato, 1968).

For immunofluorescent localization of rhodopsin, enucleated eyes were cleaned from extraocular tissue, drop fixed in $4 \%$ paraformaldehyde in PBS overnight at $4^{\circ} \mathrm{C}$, washed with PBS and embedded into paraffin at Histology Tech Services. Four-micrometer-thick paraffin sections were cut through the superior-inferior line through the optic nerve, collected on slides, and stored at room temperature until used. Immediately before immunolocalization analysis, sections were deparaffinized using Histoclear II Reagent (HS-202, National Diagnostics), rehydrated, permeabilized with $0.3 \%$ Triton-100 in PBS, blocked with $3 \%$ donkey 
serum in PBS, probed with primary anti-rhodopsin antibody, rinsed with PBS, stained with secondary antibody and Hoechst (1:1000), rinsed with PBS, mounted with mounting media (Immu-Mount, ThermoFisher Scientific) under coverslips (Fisherbrand, 12541B) and visualized with an Al confocal microscope (Nikon).

The TUNEL (terminal deoxynucleotidyl transferase dUTP nick end labeling) assay was performed on deparaffinized retinal sections using the Fluorescein In Situ Cell Death Detection Kit (11684795910, Roche) following the manufacturer's instructions. Results were expressed as an average number of TUNEL-positive nuclei per retinal section. Sections prepared from both retinas of two animals (1 male and female) of each genotype were analyzed.

Western blotting. Retinas from 5-week-old mice were collected between 12:00 and 2:00 P.M., carefully dissected, snap-frozen in liquid nitrogen and stored at $-80^{\circ} \mathrm{C}$ until used. Samples for Western blotting were obtained by solubilizing one mouse retina by sonication in $120 \mu \mathrm{l}$ of $1 \%$ Triton X-100 in PBS supplemented with protease inhibitor cocktail (Roche). Total protein concentration was measured using the DC Protein Assay kit (Bio-Rad) and samples were diluted with SDS-PAGE sample buffer to achieve a protein concentration of $1 \mathrm{mg} / \mathrm{ml}$. A small portion of each sample was used to analyze rhodopsin, whereas the rest of the sample was boiled for $5 \mathrm{~min}$ before analysis. $0.5 \mu \mathrm{g}$ of total protein was loaded by SDS-PAGE gel for rhodopsin and $20 \mu \mathrm{g}$ for other proteins. Protein bands were visualized and quantified using the Odyssey Infrared Imaging System (LI-COR Biosciences).

Rod outer segment isolation. Retinas from six 5-week-old mice of each genotype were extracted and flash-frozen. Outer segments were isolated from pooled retinas using a procedure described by Tsang et al. (1998) with slight modifications. Combined retinas were thawed $200 \mu \mathrm{l}$ of buffer containing $20 \mathrm{~mm}$ HEPES, pH 7.4, $100 \mathrm{~mm} \mathrm{KCl,} 2 \mathrm{~mm} \mathrm{MgCl}_{2}$, and 1 mM EDTA supplemented with $27 \%$ sucrose. Samples were vortexed for $30 \mathrm{~s}$ at maximum speed and spun down at for $30 \mathrm{~s}$ at $200 \mathrm{rcf}$ on a tabletop Eppendorf centrifuge. The supernatant was removed and placed on ice in a $11 \times 34 \mathrm{~mm}$ centrifuge tube (Beckman Coulter). Another $200 \mu \mathrm{l}$ of the same buffer with $27 \%$ sucrose was added to the remaining pellet and the vortexing/spinning sequence was repeated. This second supernatant was combined with the first and the total volume was then brought to $800 \mu \mathrm{l}$. This solution containing $27 \%$ sucrose was then underlaid with $1 \mathrm{ml}$ of the same buffer containing $32 \%$ sucrose. Tubes were transferred to a TLS55 swinging-bucket rotor and spun at $25,000 \mathrm{rpm}$ for $20 \mathrm{~min}$ at $4^{\circ} \mathrm{C}$ in an Optima Max-e ultracentrifuge (Beckman Coulter). Approximately 200 $\mu \mathrm{l}$ material representing the outer segment band formed at the $27-32 \%$ sucrose interface was removed and transferred to a $1.5 \mathrm{ml}$ specialized low-retention Eppendorf tube (catalog \#022363204) on ice. The fraction was diluted by $1 \mathrm{ml}$ of the same buffer without sucrose and spun down at 19,000 rpm in a Beckman-Coulter Avanti J25 ultracentrifuge for $30 \mathrm{~min}$ at $4^{\circ} \mathrm{C}$. The supernatant was then discarded and the pellet resuspended solubilized in $30 \mu \mathrm{l}$ buffer without sucrose. Protein concentration was measured using the Bradford assay and the rest used for lipid analysis.

$R N A$-seq. For whole-retina sequencing, total RNA was prepared from the retinas of three $M f_{s} d 2 a^{-/-}$(all females) and $M f_{s} d 2 a^{+/+}$( 2 males and 1 female) mice of 5-7 weeks of age using the RNeasy Mini Kit (Qiagen) with DNase digestion (RNase-Free DNase Set, Qiagen). Retinas were collected between 1:00 and 2:00 P.M. from mice killed with isoflurane, carefully dissected under a microscope and snap-frozen in liquid nitrogen. The same procedure was used to prepare retinas from two Rho ${ }^{P 23 H / W T}$ mice ( 1 male and 1 female) and their WT littermates ( 1 male and 1 female) at P33. Libraries were prepared and sequenced at GENEWIZ. FASTQ files were aligned to mouse genome GRCm38.p3 using DNAStar software v15.3.0 and normalized by either reads per kilobase of transcript (RPKM) or using the DeSeq2 method. Genes expressed at the level exceeding 1 RPKM or exceeding the linear threshold of 1 in DeSeq2 normalization in all samples were filtered out in ArrayStar. Genes that changed for $>15 \%$ with $p<0.05$ (as determined using either two-tailed homoscedastic $t$ test for RPKM normalization or $p$ value for DeSeq2 normalization) were considered differentially expressed. Foldchanges in the expression of genes were calculated based on the mean values for $M f_{s} d 2 a^{-1-}$ and WT littermates. Changes in expression levels of representative genes were plotted in GraphPad as percentage of average values for WT littermate mice calculated from datasets normalized by RPKM. A list of differentially expressed genes in $M f_{s} d 2 a^{-/-}$mice and selected genes from $R h o^{P 23 H / W T}$ mice is presented in Fig. 10-1, available at https://doi.org/10.1523/JNEUROSCI.1142-19.2019.f10-1.

To establish cellular pathways affected by Mfsd2a loss, differentially expressed genes listed in Figure 10-1 were analyzed with the Ingenuity Pathway Analysis Software (v47547484, Qiagen) using the default parameters. Enriched pathways with $p<0.05$ are listed in Fig. 10-2, available at https://doi.org/10.1523/JNEUROSCI.1142-19.2019.f10-2.

Single-cell sequencing analysis was performed using 8-week-old albino mice on the BALB/cJ, background (Jackson Laboratories, stock $\# 000651$ ) exposed to 2000 lux white light for $4 \mathrm{~h}$. The mice were killed by $\mathrm{CO}_{2}$ followed by cervical dislocation. The retinas were quickly collected for immediate dissociation using reagents and protocols of the Papain Dissociation System (Worthington Biochemical). Retinas were placed in the pre-equilibrated papain solution $[20 \mathrm{U} / \mathrm{ml}$ papain in $1.0 \mathrm{~mm}$ [sCAP] L-cysteine with 0.5 mM EDTA and $0.005 \%$ DNase I in Earle's balanced salt solution (EBSS)] and incubated in a $37^{\circ} \mathrm{C}$ water bath for $30 \mathrm{~min}$, gently mixing every 5-10 min, to break down the tissue into a single-cell suspension. The suspension was triturated by gentle pipetting and any remaining tissue clumps were allowed to settle at the bottom of the tube. The suspended cells were then transferred to a new tube and pelleted using a swinging bucket centrifuge at $300 \mathrm{rcf}$ for $5 \mathrm{~min}$ at room temperature. The papain solution was removed, and the cells were gently resuspended in pre-equilibrated ovomucoid-albumin protease inhibitor solution and DNase (2000 U/ml in EBSS). To remove cell fragments, a discontinuous density gradient was prepared by carefully layering the cell suspension on top of pre-equilibrated EBSS containing papain inhibitors ( $10 \mathrm{mg} / \mathrm{ml}$ ovomucoid and $10 \mathrm{mg} / \mathrm{ml}$ albumin) and centrifuging at $70 \mathrm{rcf}$ for $6 \mathrm{~min}$ at room temperature. The supernatants were removed and the cells were washed by gently resuspending in cold Dulbecco's PBS (DPBS) and centrifuging at $300 \mathrm{rcf}$ for $5 \mathrm{~min}$. For the second wash, the cells were resuspended in DPBS and gently passed through a $40 \mu \mathrm{m}$ filter before centrifuging at $300 \mathrm{rcf}$ for $5 \mathrm{~min}$. The pelleted cells were fixed by gently resuspending in DPBS, followed by the addition of cold methanol $(90 \%$ final concentration) dropwise to the cells, vortexing at the lowest speed setting to avoid cell clumping. The cells were incubated on ice until fixation of all cells was complete $(\sim 15 \mathrm{~min})$. Cell viability and counts were assessed before and after methanol fixation using Trypan blue on both the Countess II FL Automated Cell Counter (Life Technologies) and manually using a hemocytometer. Samples were stored at $-80^{\circ} \mathrm{C}$ overnight before proceeding with the rehydration process. Libraries were prepared using the 10X Genomics Single Cell 3'/5' RNAseq Library Prep kit (v2 Chemistry) and sequenced on the Illumina NextSeq 500 at UF Interdisciplinary Center for Biotechnology Research core facility. Datasets were processed with the 10X Chromium Cell Ranger pipeline using default parameters, and further analyzed using the 10X Chromium Loupe Browser.

Annotation of lipid classes and fatty acids. PAs, Phosphatidic acids; PEs, phosphatidylethanolamines; PE O-s, 1-O-alkyl-2-acylglycerophosphoethanolamines; LPEs, lysophosphatidylethanolamines; PSs, phosphatidylserines; LPSs, lysophosphatidylserines; PCs, phosphatidylcholines; PGs, phosphatidylglycerols; PC O-s, 1-O-alkyl-2-acylglycerophosphocholines; SMs, sphingomyelins; PIs, phosphatidylinositols; LPIs, lysophosphatidylinositols; TGs, triacylglycerols; DGs, diacylglycerols; Cers, ceramides; CLs, cardiolipins; GalCers, galactosylceramides; CFs or Chols, cholesterols; CEs, cholesterol esters. Lyso- and phospholipid species were annotated using the total number of carbon atoms and total number of double bonds in fatty acid moieties. DHA (22:6n-3); DPAn-6, docosapentaenoic acid (22:5n-6); AA (20:4n-6); VLCFA, very long fatty acid, defined in this study as one containing 24 and more carbons; MUFA, monounsaturated fatty acid (e.g., 16:1, 18:1, 20:1); SFA, saturated fatty acid (e.g., 16:0, 18:0, 20:0).

Quantitative shotgun lipidomics. Eyes were collected from mice transcardially perfused with $30 \mathrm{ml}$ of PBS. Retinas were extracted in Hank's solution, collected in $2.0 \mathrm{ml}$ Eppendorf tubes (\#0030 120.094), topped off with nitrogen, flash frozen in liquid nitrogen, stored at $-80^{\circ} \mathrm{C}$, and transported on dry ice. Retinas were collected from three $M f s d 2 a^{-/-}$(2 fe- 
A
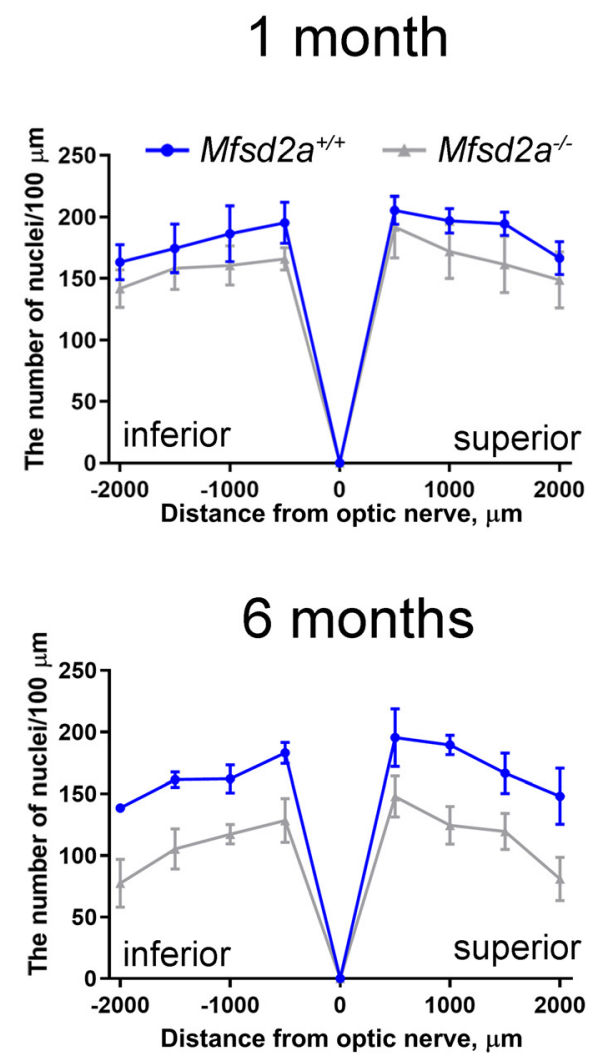

B
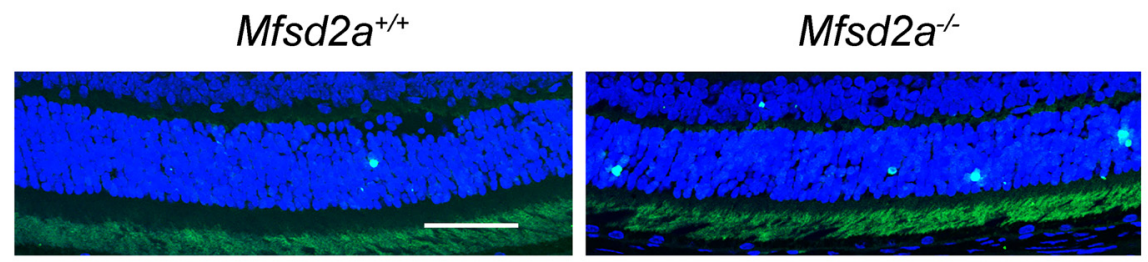
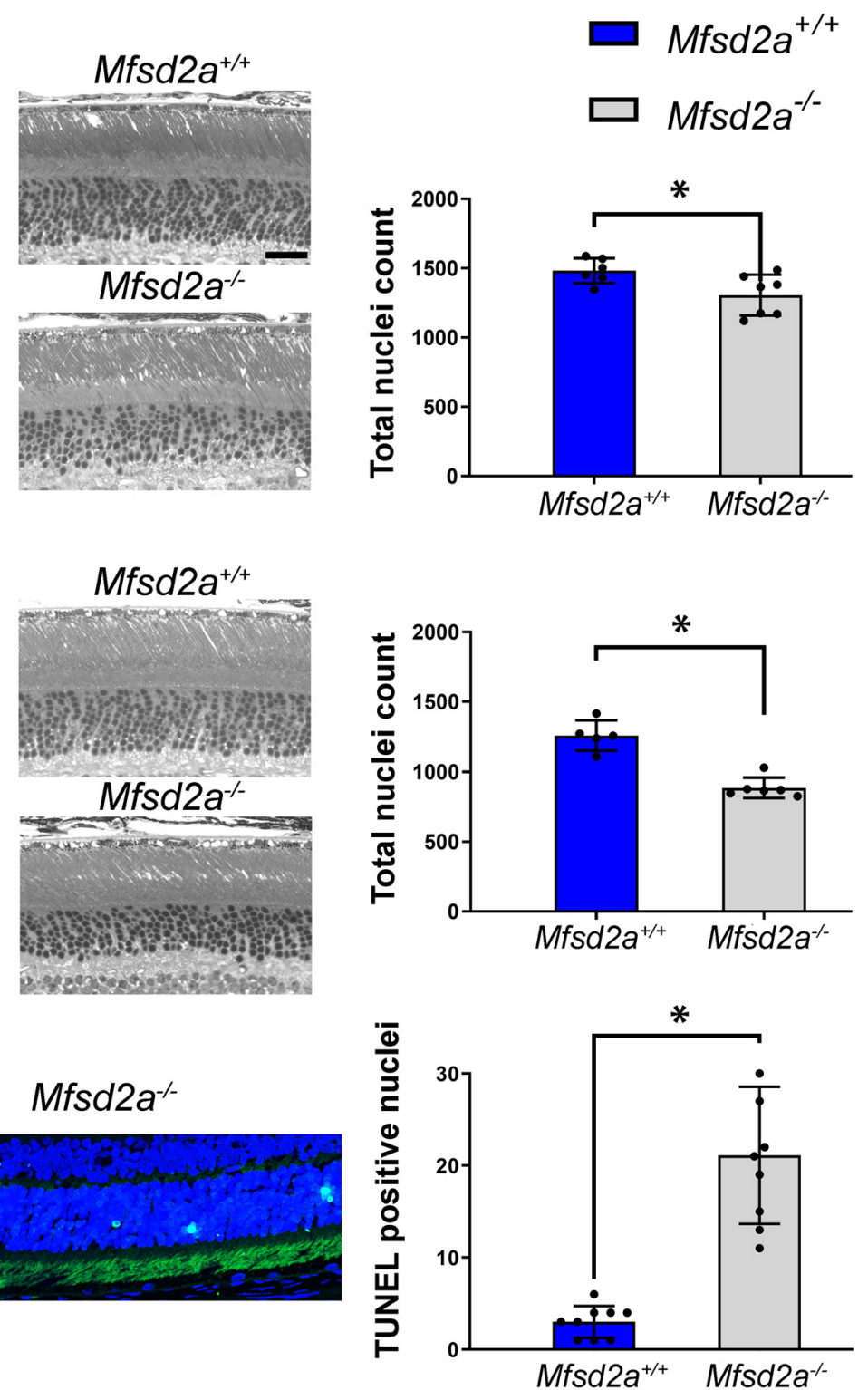

Figure 1. Morphometric analysis of photoreceptors in $\mathrm{Mfs}_{2} 2 a^{-/-}$mice. $\boldsymbol{A}$, Left, Spider diagrams representing the number of photoreceptor nuclei in $100 \mu \mathrm{m}$ segments of the inferior and superior retina counted at various distances from the optic nerve head. Data collected from 1-and 6-month-old Mfsd $2 a^{-/-}$mice and their WT littermates are shown as mean \pm SD; ${ }^{*} p \leq 0.05$. The number of eyes analyzed at 1 month was $7 \mathrm{Mfsd} 2 a^{-1-}$ and $6 \mathrm{WT}$; at 6 months $6 \mathrm{Mfsd}_{2 a^{-1-}}$ and $5 \mathrm{WT}$. Mouse genotypes are indicated above the panels. Middle, Representative images of superior retinal cross-sections at $\sim 1 \mathrm{~mm}$ distance from the optic nerve head. Scale bar, $25 \mu \mathrm{m}$. For images of representative cross-sections through the entire retinas see Figure 2 . Right, The total number of nuclei in all $100 \mu \mathrm{m}$ retinal segments presented in the spider diagrams. $p=0.028$ for 1-month-old mice; $p=7.7 \times 10^{-5}$ for 6-month-old mice. $\boldsymbol{B}$, Left, Representative images of TUNEL-processed retinas from Mfsd $2 a^{-/-}$and WT littermate mice analyzed at 1 month of age. The green fluorescent signal represents nuclei of apoptotic cells. Right, An average number of TUNEL-positive cells per whole cross-section of Mfsd $2 a^{-/-}$and WT mice. At least four independently processed sections from both eyes of two mice (1 male and 1 female) for each genotype were analyzed. Data are shown as mean $\pm S D ; p=2 \times 10^{-6}$.

males and 1 male) and three $M f_{s} d 2^{+/+}$(2 males and 1 female) mice at P38-P40.

Before lipid extraction, two retinas were homogenized at $4^{\circ} \mathrm{C}$ in $250 \mu \mathrm{l}$ of isopropanol using $1 \mathrm{~mm}$ zirconium beads (BioSpec, 11079110z) on a TissueLyser (Qiagen, 85300). Retina and outer segment membrane preparations were dried under vacuum and lipids were extracted with $1.4 \mathrm{ml}$ or $0.7 \mathrm{ml}$, respectively, of methyl tert-butyl ether (MTBE)/MeOH (10:3; v:v) spiked with internal lipid standards. The internal standard mixture for retinal samples contained the following: $1.59 \mathrm{nmol} \mathrm{CF} \mathrm{D}_{7}, 1.81 \mathrm{nmol}$ $\mathrm{CE} \mathrm{D}_{7}$ 16:0, $1.49 \mathrm{nmol} \mathrm{TG} \mathrm{D}_{5}$ 50:0, $1.04 \mathrm{nmol} \mathrm{DG} \mathrm{D}_{5}$ 34:0, $1.47 \mathrm{nmol} \mathrm{PC}$ 25:0, $1.94 \mathrm{nmol}$ LPC 13:0, $0.48 \mathrm{nmol}$ PS 25:0, $1.05 \mathrm{nmol}$ PE 25:0, 1.52 nmol LPE 13:0, $0.86 \mathrm{nmol}$ PI 25:0, $0.11 \mathrm{nmol}$ LPI 13:0, $0.49 \mathrm{nmol}$ PG 25:0, 1.3 nmol Cer 30:1, 0.48 nmol SM 30:1, 0.49 nmol GalCer 30:1, 0.55 nmol PA 25:0. The internal standard mixture for outer segment membranes contained $1.78 \mathrm{nmol} \mathrm{CF}_{7}, 2.21 \mathrm{nmol} \mathrm{CE} \mathrm{D}_{7} 16: 0,1.04 \mathrm{nmol} \mathrm{TG}$
$\mathrm{D}_{5}$ 50:0, $0.6 \mathrm{nmol}$ DG $\mathrm{D}_{5}$ 34:0, $1.38 \mathrm{nmol}$ PC 25:0, 0.39 nmol LPC 13:0, $0.14 \mathrm{nmol}$ PS 25:0, $0.59 \mathrm{nmol}$ PE 25:0, $0.09 \mathrm{nmol}$ LPE 13:0, $0.48 \mathrm{nmol}$ PI 25:0, 0.06 nmol LPI 13:0, 0.14 nmol PG 25:0, 0.07 nmol Cer 30:1, 0.48 nmol SM 30:1, $0.27 \mathrm{nmol}$ GalCer 30:1, 0.13 nmol PA D7 33:0. Next, 280 $\mu \mathrm{l}$ (retina) or $140 \mu \mathrm{l}$ (outer segments) of water was added and the mixture incubated in an Eppendorf mixer for $1.5 \mathrm{~h}$ at $4^{\circ} \mathrm{C}$.

Phase separation was induced by centrifugation at $13,400 \mathrm{rpm}$ for 30 min at $4^{\circ} \mathrm{C}$ on a benchtop centrifuge. The organic phase was transferred into glass vial and stored at $-20^{\circ} \mathrm{C}$ until analyzed. Three hundred microliter aliquots of the extract were dried under vacuum, resuspended in 50 $\mu \mathrm{l}$ of MTBE/ methanol/ water (10:3:1; v:v:v) and subjected to thin-layer chromatography (Macherey-Nagel, Nano-SILGUR-20; $10 \times 10 \mathrm{~cm}$ ) using a mobile phase chloroform/ethanol/water/trimethylamine (TEA; 30 : 35:7:35; v:v:v:v). Three fractions were collected by scraping the silica layer and lipids re-extracted with $\mathrm{MTBE} / \mathrm{methanol}$ as described above, but 


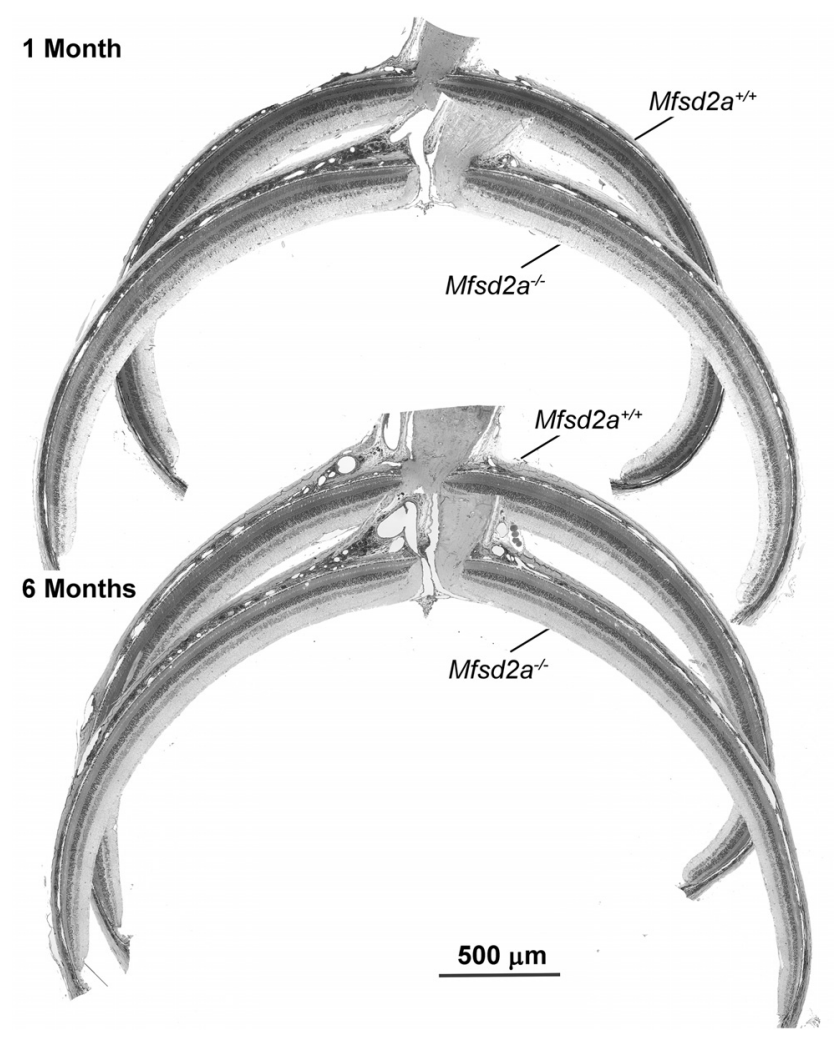

Figure 2. Plastic sections cut through the entire retinas of $M f s d 2 a^{-/-}$mice and their WT littermates at indicated ages. Sections are stained by toluidine blue and shown in grayscale.
A

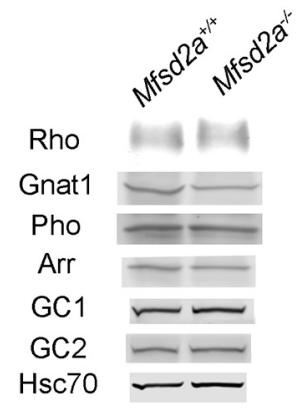

B

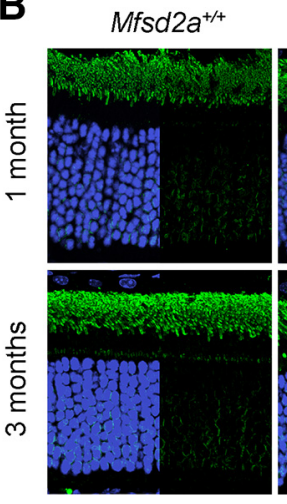

Mfsd2a-r

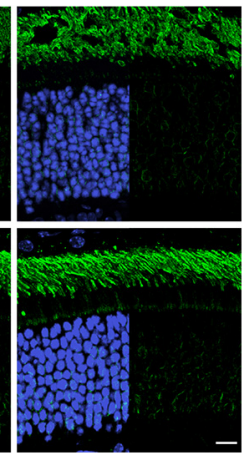

Figure 4. Loss of Mfsd2a does not affect expression levels and localization of phototransduction proteins. $A$, The expression of rhodopsin and several other major photoreceptor proteins in the retinas of 5-week-old Mfsd2a $a^{-1-}$ mice. Western blots were performed with aliquots of retinal lysates containing $0.5 \mu \mathrm{g}$ total protein for rhodopsin and $20 \mu \mathrm{g}$ total protein for other proteins. Each determination was repeated for at least four pairs of WT and Mfsd2a $a^{-/-}$animals. Rho, Rhodopsin; Gnat1, transducin $\alpha$-subunit; Pho- phosducin; Arr, arrestin-1; $\mathrm{GC} 1$ and GC2, two retinal guanylate cyclase is oforms. Hsc70 is used as a loading control. $B$, Subcellular localization of rhodopsin (green) in 1-and 3-month-old Mfsd $2 a^{-1-}$ and their WT littermate mice. Scale bar, $10 \mu \mathrm{m}$. Nuclei are stained by Hoechst (blue).

without adding internal standards. Finally, $1.2 \mathrm{ml}$ of organic extracts from each fraction were collected and stored in glass vials at $-20^{\circ} \mathrm{C}$. For mass spectrometric analysis, aliquots of collected fractions were dried and re-dissolved in isopropanol/methanol/chloroform mixture (4:2:1; v:v:v) with $7.5 \mathrm{~mm}$ ammonium formate or in ethanol/chloroform 5:1 (v:v) mixture with $0.1 \%$ TEA. $170 \mu$ l of the fraction $1(\mathrm{Rf}=0.5-1.0)$ were dissolved in $180 \mu \mathrm{l}$ ammonium formate or the TEA mix, $80 \mu \mathrm{l}$ fraction 2 $(\mathrm{Rf}=0.24-0.5)$, in $200 \mu \mathrm{l}$ of the TEA mix, and $80 \mu \mathrm{l}$ of fraction $3(\mathrm{Rf}=$ $0-0.24)$ in $200 \mu \mathrm{l} \mathrm{NH}_{4} \mathrm{HCO}_{2}$ or the TEA mix.

The fractions were analyzed on a hybrid quadrupole, Orbitrap tandem mass spectrometer Q Exactive (ThermoFisher Scientific), equipped with a robotic nanoflow ion source TriVersa NanoMate (Advion BioSciences) using $4.1 \mu \mathrm{m}$ na-

A

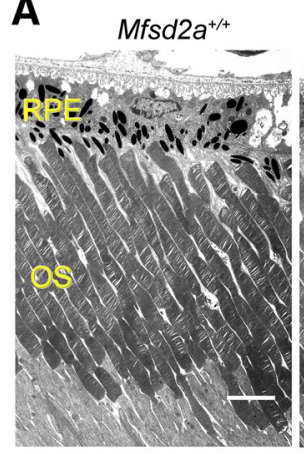

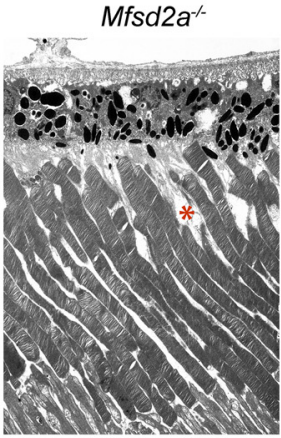

B

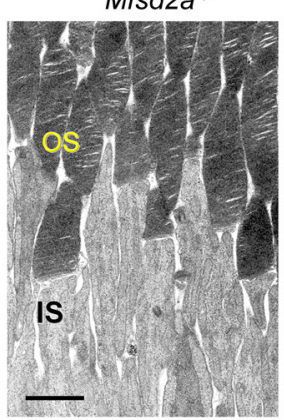
noelectrospray chips. The ion source was controlled by the Chipsoft 8.3.1 software (Advion BioSciences). Ionization voltage and back pressure were set to $\pm 0.96 \mathrm{kV}$ and 1.25 psi for mixtures containing ammonium formate and $-2.0 \mathrm{kV}$ and $1.0 \mathrm{psi}$ for mixtures containing TEA. The temperature of the ion transfer capillary was $200^{\circ} \mathrm{C}$; S-lens RF level was set at $50 \%$. For lipidomic measurements FT MS spectra in positive or negative mode were acquired within the $m / z$ range of $400-1200$ at the target mass resolution of $R_{m / z 200}=140,000$; automated gain control (AGC) was set at $3 \times 10^{6}$ and the maximum injection time at $3000 \mathrm{~ms}$. Subsequently, HCD FT MS/MS spectra were acquired at the same mass resolution using an inclusion list of $m / z$ of lipids recognized in FT MS spectra. Precursor isolation window was 1.0 Da; AGC was set at $2 \times 10^{4}$ and maximum injection time at $650 \mathrm{~ms}$. Normalized collision energies ( $15 \%$ in positive mode and $25 \%$ in negative mode) were used to confirm identity of lipids. Cholesterol was quantified as described by Sales et al. (2016). Acquired spectra were filtered by PeakStrainer (https://git.mpi-cbg.de/labShevchenko/PeakStrainer/wikis/home; Schuhmann et al., 2017) and lipids were identified and quantified by LipidXplorer software (Herzog et al., 2011) considering better than 5 ppm mass accuracy and signal-to-noise ratio above

Figure 3. Ultrastructural analysis of Mfsd $2 a^{-/-}$mice. $A$, Electron micrographs of retinal cross-sections from 1-monthold mice of each genotype. A malformed outer segment (OS) in the Mfsd $2 a^{-/-}$retina is marked by an asterisk. Scale bar, $5 \mu \mathrm{m}$. B, A higher-magnification view of the inner segment (IS)- outer segment interface. Scale bar, $2 \mu \mathrm{m}$. C, A highermagnification view of the outer segment-RPE interface. Scale bar, $1 \mu \mathrm{m}$. D, A microglial cell in the subretinal space of the Mfsd $2 a^{-1-}$ mouse (red arrow). Note a nucleus on the left side and a dense cluster of phagosomes on the right side of this cell. Scale bar, $1 \mu \mathrm{m}$. 

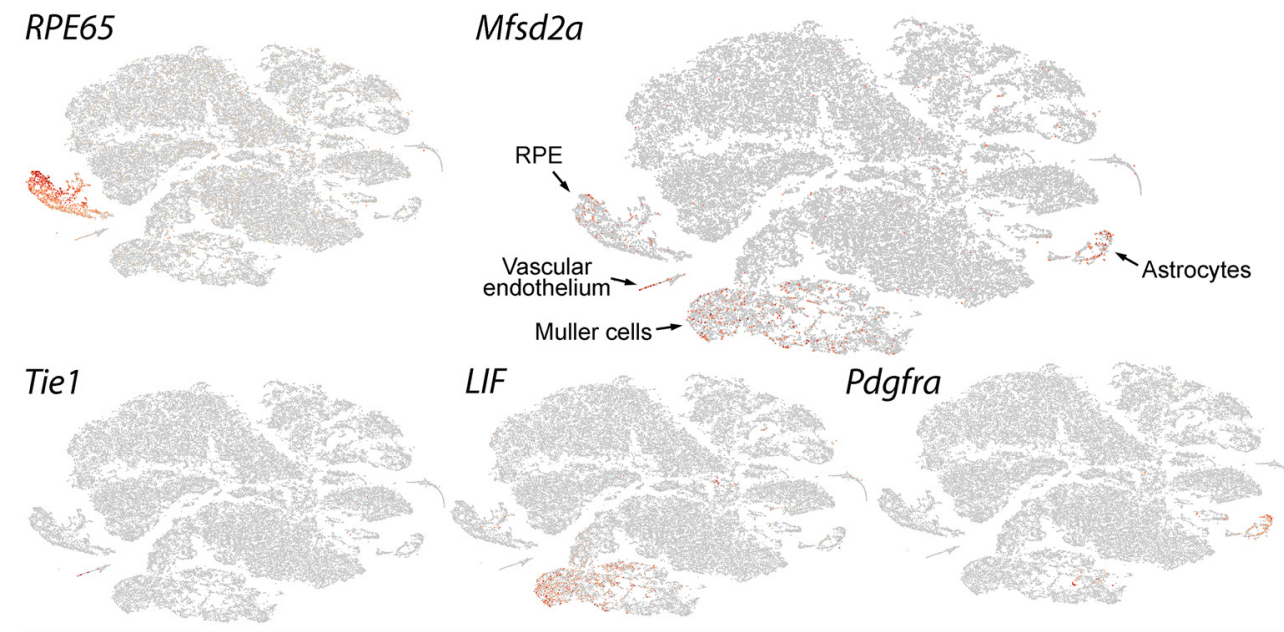

Figure 5. T-distributed stochastic neighbor embedding (t-SNE)-dimension reduction of gene expression profiles of single cells prepared from WT mouse eyes. The populations of cells expressing Mfsd2a overlap with cells expressing markers of RPE (Rpe65, retinal pigment epithelium-specific 65 kDa protein), vascular endothelium cells (Tie1, tyrosine kinase with lg-like and EGF-like domains 1), Müller cells (LIF, leukemia inhibitory factor), and astrocytes (Pdgfra, platelet derived growth factor receptor $\alpha$ ).
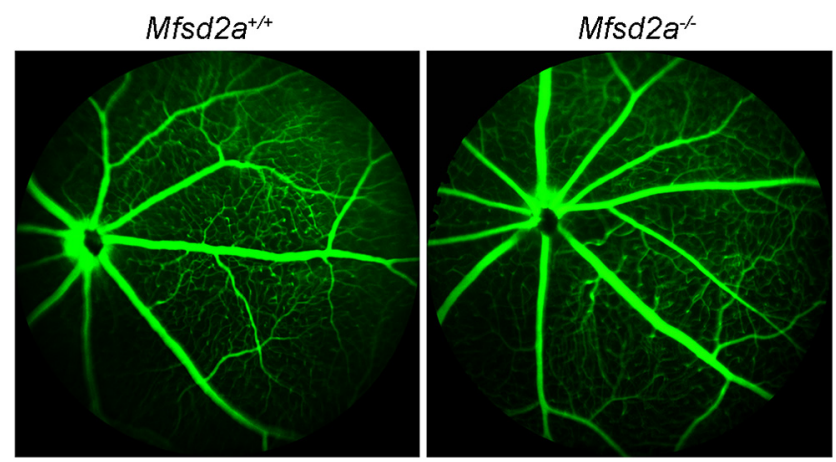

Figure 6. Fluorescein angiograms of Mfsd $2 a^{-/-}$and WT littermate mice. The data are taken from one of four similar experiments (see Materials and Methods).

the value of 3.0. Cardiolipins were quantified using the PG standard. CE, TG, GalCer, and PA were detected at trace amounts and were not quantified.

To estimate changes in the amounts of SFA, MUFA, DHA, AA, and VLCFA (defined as having 24 or more carbons) in whole retinas shown in Figure $7 D$, we selected lysophospholipids and phospholipids containing these fatty acids based on previous fragmentation studies performed with rat retinas (Schuhmann et al., 2011). The relative amounts of fatty acids of each type were determined by taking into consideration the presence of two fatty acids in phospholipids and one fatty acid in lysophospholipids. Results were expressed as percentage of total fatty acids incorporated into all measured lysophospholipids and phospholipids.

Changes in the fraction of outer segment phospholipids containing MUFA, DHA, and AA (see Fig. 9B) were calculated from molar fractions of major PC, PE, PS, and PI species containing fatty acid of each type and shown as percentage of total phospholipids. Phospholipids used in these calculations are highlighted on Fig. 7-1, available at https://doi.org/ 10.1523/JNEUROSCI.1142-19.2019.f7-1 (tabs "Major OS PL \%" and "Major retinal PL \%").

Fundus fluorescein angiography. Mice were anesthetized with a mixture of ketamine-xylazine (95-9 $\mathrm{mg} / \mathrm{kg}$, respectively) and their eyes were dilated with ophthalmic solutions of atropine (1\%) and phenylephrine $(2.5 \%)$. Mice were administered an intraperitoneal injection of 100 $\mathrm{mg} / \mathrm{kg}$ fluorescein $(\sim 0.02 \mathrm{ml}$ of $100 \mathrm{mg} / \mathrm{ml}$ fluorescein). Images were acquired starting $1 \mathrm{~min}$ post-injection using a Micron IV fundus camera. Four $M f s d 2 a^{-1-}$ and their WT littermates were analyzed at 6 weeks of age. Experiments were performed between 10:00 A.M. and 1:00 P.M.
Suction electrode recordings. Suction electrode recordings from the outer segments of intact mouse rods were performed as previously described (Gross and Burns, 2010). Dark-adapted retinas were stored on ice in L-15 supplemented with $10 \mathrm{~mm}$ glucose. Recordings were performed in oxygenated, bicarbonate buffered Locke's solution supplemented with $10 \mathrm{~mm}$ glucose at $35-37^{\circ} \mathrm{C}$. The suction pipette contained HEPESbuffered Locke's solution, $\mathrm{pH}$ 7.4, connected to a calomel electrode via an agar bridge. An agar bridge also connected the bath to the opposite calomel half-cell.

Brief (10 ms, $500 \mathrm{~nm}$ ) flashes of calibrated strength were used to elicit electrical responses, which were amplified (AxoPatch 200B, Molecular Devices), filtered at $30 \mathrm{~Hz}$ with an 8-pole Bessel (Frequency Devices), and digitized at $200 \mathrm{~Hz}$ using custom-written acquisition procedures in IgorPro (WaveMetrics). Responses to a large number $(>30)$ of dim flashes were averaged and used to determine the mean time to peak and integration time (time integral of the response divided by the peak amplitude) if the peak amplitude was $<20 \%$ of the maximal response amplitude (dark current). Variance to mean analysis of the dim flash responses was used to calculate the average single photon response for each cell, and saturating flash responses were used to calculate the dominant time constant of recovery $\left(\tau_{\mathrm{D}}\right)$ as previously described (Krispel et al., 2006; Gross and Burns, 2010). Statistical tests for differences between genotypes were determined for the population average kinetic parameters using a two-tailed $t$ test assuming equal variances. Error bars represent SEM.

Statistical analysis. Statistical analyses were performed in GraphPad Prism 5 software or Excel 2016. Results are expressed as the mean \pm SD, unless noted otherwise. Differences are considered significant if $p \leq 0.05$, as determined by a two-tailed homoscedastic $t$ test; n.s. indicates no significant difference $(p>0.05)$.

\section{Results}

Slow photoreceptor degeneration in $M f s d 2 a^{-/-}$mice

We conducted morphometric analysis of $M f_{S} d 2 a^{-1-}$ retinas in thin plastic sections at 1 and 6 months of age. The number of photoreceptors was reduced by 12 and $30 \%$, respectively, compared with WT littermates of the same ages (Figs. 1A,2). The ongoing cellular loss in mutant mice was corroborated by an $\sim$ sevenfold increase in the number of apoptotic nuclei in the outer nuclear layer detected by the TUNEL assay (Fig. 1B). The progressive nature of photoreceptor loss and the presence of apoptotic nuclei argue that the reduction in the photoreceptor cell count in young $M f_{s} d 2 a^{-/-}$mice indeed reflects ongoing photoreceptor degeneration rather than some form of developmental deficiency. 


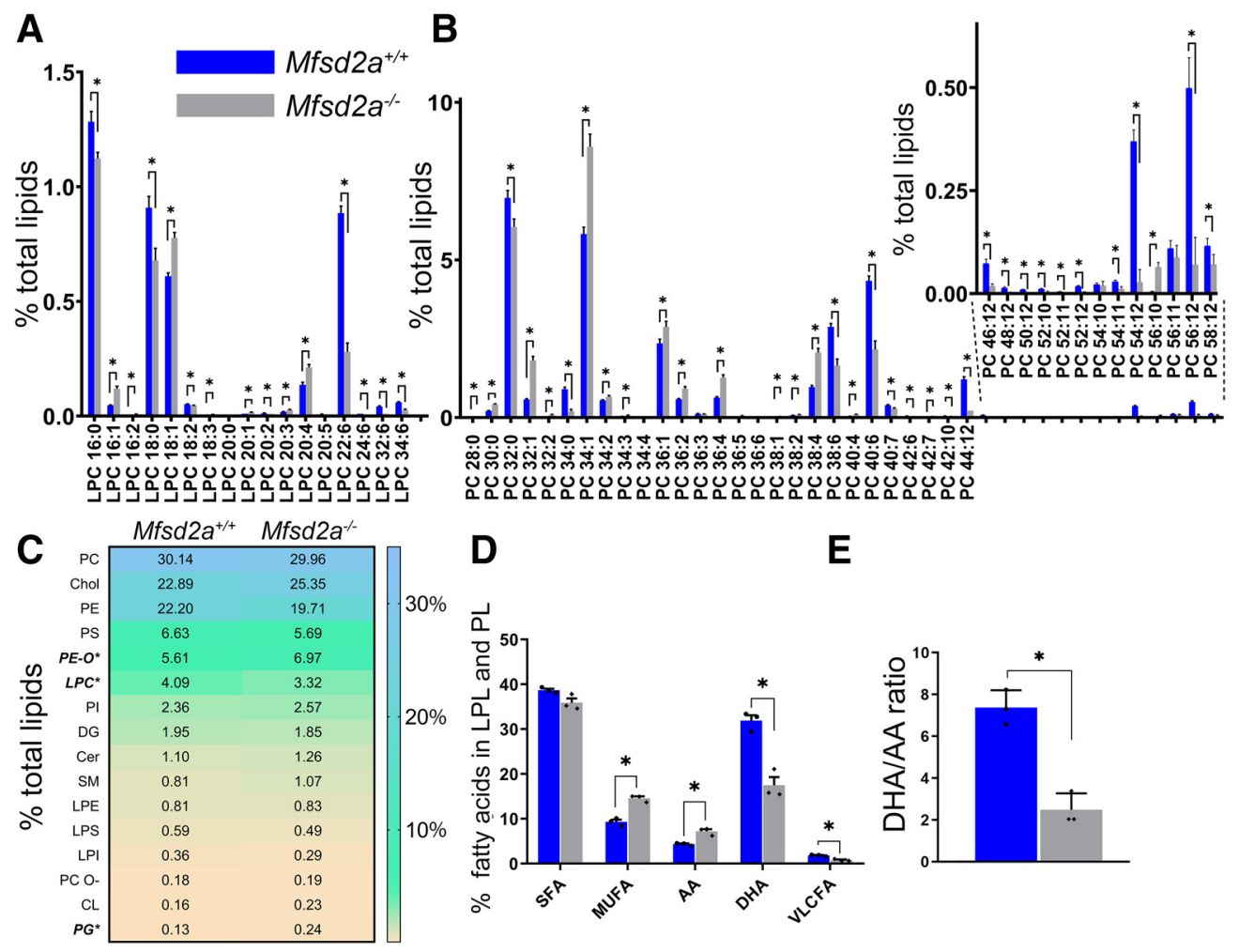

Figure 7. Lipidomic analysis of isolated retinas from Mfsd2a ${ }^{-/-}$mice and their WT littermates. Lipid profiles for $(\boldsymbol{A})$ lysophosphatidylcholine and $(\boldsymbol{B})$ phosphatidylcholine shown as percentage of total amount from measured lipid classes (for complete dataset, see Figure 7-1, available at https://doi.org/10.1523/JNEUROSCI.1142-19.2019.f7-1). Statistically significant changes ( $p \leq 0.05$ ) are marked by asterisks. $C$, Relative representation of all measured lipid classes shown as percentage total. Three lipid classes for which the change was statistically significant between the two mouse types are marked with asterisks. The abundance of each lipid class in each dataset is color-coded with the scale shown on the right. $D$, Changes in the fractions of SFA, MUFA, AA, DHA, and VLCFA in Mfsd $2 a^{-1-}$ and WT mice. Values are calculated based on the total amounts of each fatty acid type in all identified lysophospholipids (LPL) and phospholipids (PL; for complete dataset, see Figure 7-1, available at https://doi.org/10.1523/JNEUROSCI.1142-19.2019.f7-1). E, The DHA/AA molar ratios in Mfsd2a ${ }^{-/-}$and WT mice. Data are shown as mean \pm SD.

However, the EM analysis of $M f s d 2 a^{-/-}$photoreceptors did not reveal any notable abnormalities in either the overall ultrastructure of outer segments from surviving rods (Fig. $3 A$ ) or the spacing of photoreceptor discs (Fig. $3 A-C$ ). Also normal were the appearance of the active zone of new disc formation at the outer segment base and the interface between the outer segment tip and the retinal pigment epithelium (Fig. $3 B, C$ ). The only distinctions from WT retinas were as follows: (1) an occasional appearance of malformed outer segments, apparently belonging to dying $M f_{s} d 2 a^{-1-}$ photoreceptors (Fig. 3A, red asterisk); and (2) the presence of mononuclear phagocytes in the subretinal space (Fig. $3 D$ ). The latter are most likely microglia, as shown by Wong et al. (2016). No overt abnormalities in other retinal layers were observed as well. The normal appearance of outer segment ultrastructure was different from (Wong et al., 2016) who observed gross morphological abnormalities in $M f s d 2 a^{-1-}$ photoreceptors in both young and aged animals. One potential explanation is that they characterized a $M f s d 2 a^{-/-}$mouse obtained from a different source that contained the $r d 8$ mutation (D. L. Silver, personal communication), whereas our line was back-crossed to the $r d 8$-free C57BL/6J background and specifically tested for the absence of $r d 8$. It is conceivable that $r d 8$ serves as a genetic factor confounding the $M f s d 2 a^{-/-}$phenotype. We also cannot exclude the possibilities that these differences are explained by using different diets or subtle differences in tissue processing protocols.

Consistent with only a minor photoreceptor loss, we did not observe a significant reduction in the expression levels of representative phototransduction proteins, measured in whole retinal lysates of 1-month-old mice (Fig. 4A). We also assessed the local- ization of rhodopsin in rod photoreceptors of $M f_{s} d 2 a^{-1-}$ mice. Considering a previous report that rhodopsin is partially mislocalized from the outer to inner segments of $M f s d 2 a^{-/-}$rods (Wong et al., 2016), we used thin retinal sections, which aids inner segment rhodopsin detection, and optimized image contrast to visualize rhodopsin in this region. Despite these precautions, we observed no difference in inner segment rhodopsin staining between the retinas of $M f s d 2 a^{-/-}$mice and their WT littermates (Fig. $4 B$ ), indicating that rhodopsin targeting and trafficking are not affected by the Mfsd2a knock-out.

\section{$M f s d 2 a$ expression in the retina}

Previous study demonstrated robust expression of Mfsd2a in the RPE and retinal vasculature endothelium (Wong et al., 2016). We investigated whether the $M f_{s} d 2 a$ gene could be additionally expressed in other retinal cell types using single-cell transcriptomic analysis. We used a large single-cell transcriptome dataset obtained from an ongoing study of light damaged retinas. $M f s d 2 a$ transcript was detected in four clearly separated cell clusters (Fig. 5). As expected, one of them represented RPE (based on expression of the Rpe65 marker) and another represented vascular endothelial cells (based on expression of the Tiel marker). However, we also detected robust $M f s d 2 a$ expression in cell clusters expressing the Muller cell marker, LIF (Joly et al., 2008), and the astrocyte marker, Pdgfra (Mudhar et al., 1993), suggesting that Mfsd2a participates in the lipid transport across the membranes of these glial cells. Expression of $M f s d 2 a$ in retinal astrocytes is consistent with single-cell transcriptomics analysis of the brain (Vanlandewijck et al., 2018). Unfortunately, we could not iden- 
tify antibodies that could corroborate these findings by immunohistochemistry. It is also worth noting that transcriptional analysis performed in a recent study (Benedicto et al., 2017) suggested the presence of $M f_{s} d 2 a$ in the choroidal cells, which were absent in our dataset and not investigated here.

\section{Analysis of the retina-blood barrier in $M f s d 2 a^{-/-}$mice}

There has been a controversy between the studies by two other laboratories analyzing the brain phenotype of the $M f_{s} d 2 a^{-/-}$ mouse. Although Ben-Zvi et al. (2014) claimed that the major consequence of the Mfsd2a knock-out is the blood-brain barrier leakage, Wong et al. (2016) did not observe blood-retina barrier leakage in either the brain or the retina. This prompted us to interrogate whether the lack of Mfsd2a causes a disruption of the blood-retina barrier in our mouse (which was the same strain as used by Ben-Zvi et al., 2014). We used fluorescein angiography, which assesses the integrity of tight junctions in the retinal vasculature endothelium in vivo. Consistent with results reported by Wong et al. (2016), our analysis revealed no evidence of retinal vascular leakage, at least as assessed by this technique (Fig. 6).

Lipid composition of $M f s d 2 a^{-/-}$retinas We next compared the lipid composition of retinas obtained from 6-week-old $M f_{s} d 2 a^{-1-}$ and WT mice. Lipid extracts, pre-fractionated by TLC, were subjected to quantitative top-down shotgun analysis on a high-resolution LTQ Orbitrap mass spectrometer (Schuhmann et al., 2011, 2012). Peaks representing intact molecular ions of lipids were recognized by their accurately determined masses and, therefore, the detection specificity was not biased by the composition of their fatty acid moieties. Notably, alternative analytical approaches (e.g., multiple reaction monitoring) that rely on the detection of carboxylate fragments produced from molecular anions of glycerophospholipids could discriminate against PUFAcontaining and, particularly, VLCFA-containing lipid species. Unlike carboxylate fragments of saturated fatty acid moieties, the unsaturated fragments are unstable, undergo facile collisional degradation, and therefore are detected as peaks with much lower amplitudes. Using the shotgun methodology allowed us to identify multiple VLCFA species with $>30$ carbon atoms and 6 double bonds, whereas none of them were reported by Wong et al. (2016), who relied on targeted mass spectrometry.

Because Mfsd2a is the LPC transporter, we first analyzed the retinal content of this phospholipid and observed a remarkable shift in the saturation profile of its fatty acid moieties: of 17 detected LPC species, 15 were statistically significantly changed (Fig. 7A). These changes could be summarized in four groups: (1) asterisks.

\section{A Lyso- and phosphatidylethanolamines}

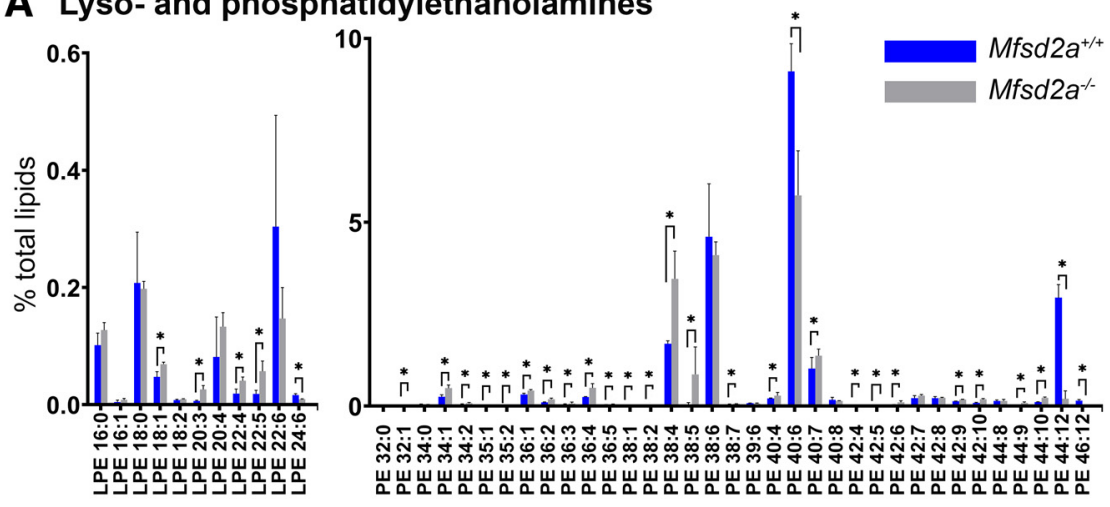

B Lyso- and phosphatidylserines

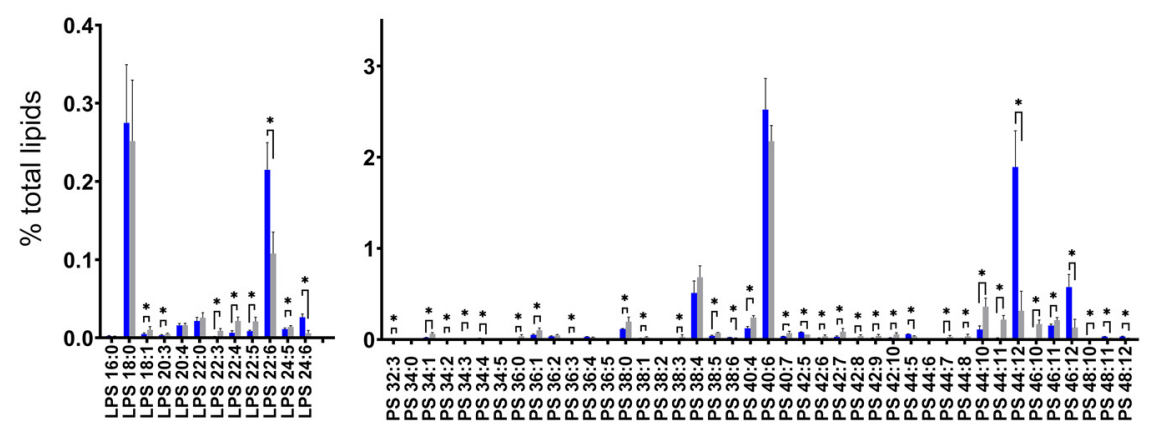

C Lyso- and phosphatidylinositols

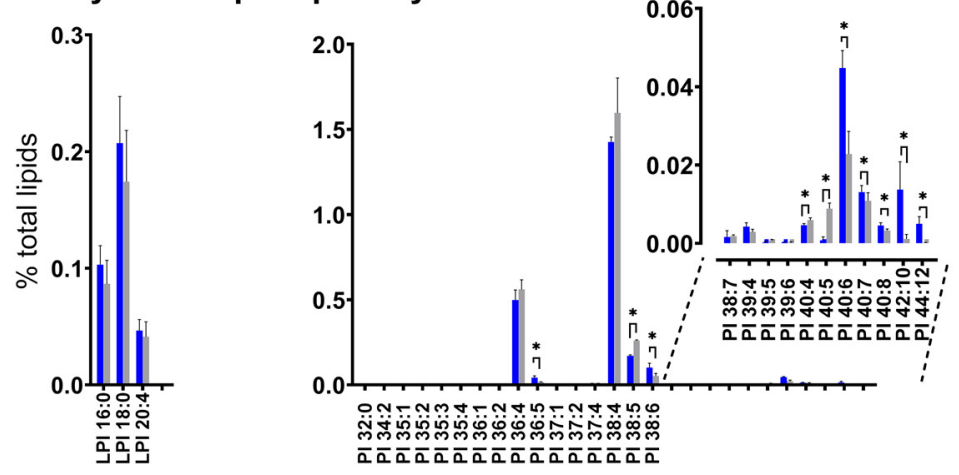

Figure 8. Lipidomic analysis of LPE, PE, LPS, PS, LPI and PI phospholipids in isolated retinas from Mfsd $2 a^{-1-}$ mice and their WT littermates. Lipid profiles of $(\boldsymbol{A})$ lyso- and phosphatidylethanolamines, $(\boldsymbol{B})$ lyso- and phosphatidylserines, and $(\boldsymbol{C})$ lyso- and phosphatidylinositols shown as percentage of total phospholipids from all measured classes (for complete dataset, see Figure 7-1, available at https://doi.org/10.1523/JNEUROSCI.1142-19.2019.f7-1). Statistically significant changes ( $p \leq 0.05$ ) are marked by

a reduction of LPC with fatty acids containing 22 and more carbons, such as LPC22:6 (containing DHA), LPC24:6, LPC32:6 and LPC34:6; (2) an increase in LPC20:4 (containing AA); (3) an increase in LPC containing MUFAs (LPC16:1, LPC18:1, LPC20: 1 ); and (4) miscellaneous changes in proportions of LPC species containing $0,2,3$ and 5 double bonds.

In a similar pattern, we observed a reduction in the major PC species containing DHA (such as PC38:6, PC40:6, and PC44:12) and those containing 24 and more carbons (VLCFA; PC46:12, PC54:12, PC56:12), as well as an increase in the fraction of PC containing AA (PC36:4, PC38:4) and MUFAs (PC32:1, PC34:1, PC36:1; Fig. 7B). This trend was present for other major phospholipid classes-PE, PS, and PI-although the extent of fatty acid moieties' remodeling was smaller than for PC (Fig. 8). 


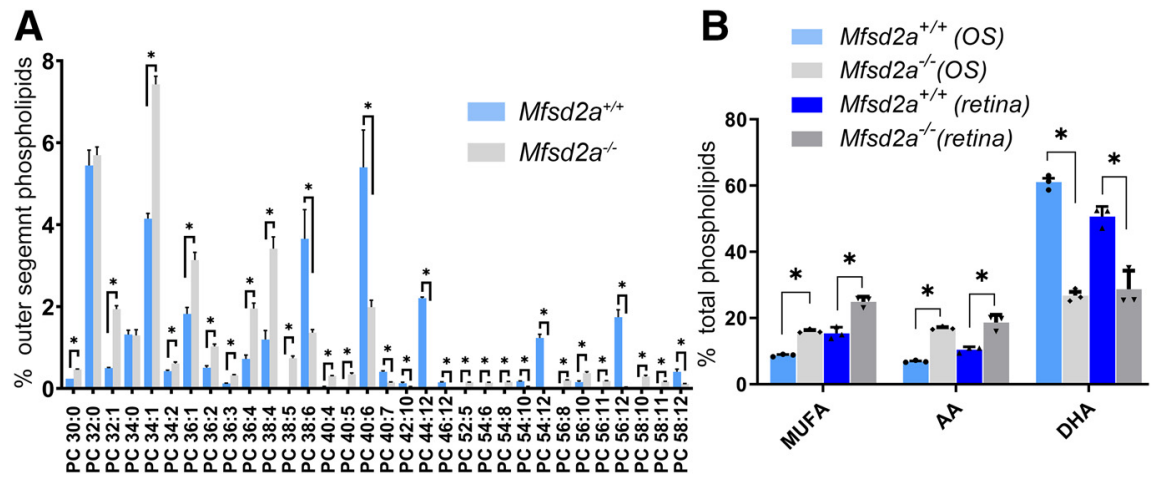

Figure 9. Lipidomic analysis of rod outer segment preparations isolated from Mfsd2a ${ }^{-/-}$mice and their WT littermates. $A$, Lipid profiles for $\mathrm{PC}$ species shown as percentage of total outer segment phospholipid content. Only phospholipids present at the levels exceeding $0.15 \%$ of the total phospholipids content in either genotype are shown. For a complete dataset, see Figure $7-1$, available at https://doi.org/10.1523/JNEUROSCI.1142-19.2019.f7-1 (tab "Major OS PL \%"). B, Changes in the fractions of phospholipids containing MUFA, AA, and DHA shown as percentage of total phospholipids. The values are calculated from molar fractions of major PC, PE, PS, and PI species containing fatty acids of each type and shown as percentage of total phospholipids (Figure 7-1, available at https://doi.org/10.1523/JNEUROSCI.1142-19.2019.f7-1, tab Major OS PL \%). For comparison, a similar calculation for PC, PE, PS, and PI only is performed for the whole retina and plotted side-by-side with outer segment data (Figure 7-1, available at https://doi.org/10.1523/JNEUROSCI.1142-19.2019.f7-1, tab “Major retinal PL\%"). Data are shown as mean \pm SD $(n=3)$. Statistically significant changes ( $p \leq 0.05)$ are marked by asterisks.

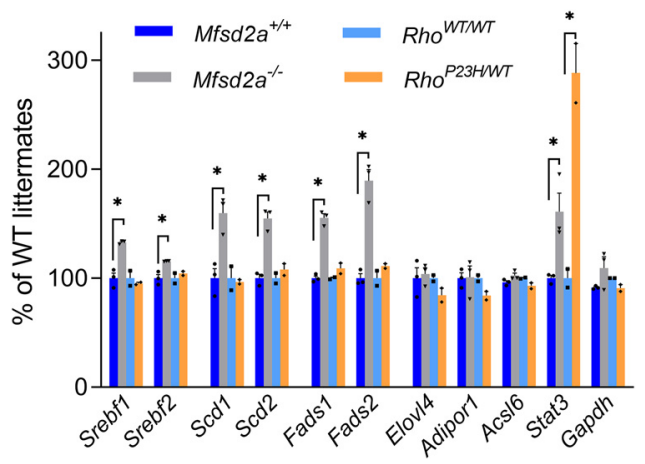

Figure 10. Comparative analysis of selected gene expression changes in Mfsd2a-/- and Rho ${ }^{P 23 H / W T}$ mice. Relative gene expression levels were calculated from the corresponding RPKM values. Data are shown as percentage of WT littermates for each genotype and expressed as mean $\pm \mathrm{SD}$ ( $n=3$ for Mfsd $2 a^{-1-} ; n=2$ for Rho ${ }^{P 23 H / W T}$ mice). Gapdh (glyceraldehyde 3-phosphate dehydrogenase) was used as a sample normalization control. Statistically significant changes ( $p \leq 0.05$ ) are marked with asterisks. For a complete dataset, see Figure 10-1, available at https://doi.org/10.1523/JNEUROSCI.1142-19.2019.f10-1, and for the enriched pathway analysis, see Figure 10-2, available at https://doi.org/10.1523/JNEUROSCI. 1142-19.2019.f10-2.

Consistent with the role of Mfsd2a in LPC transport across the blood-retina barrier, we observed an $\sim 19 \%$ reduction in the total molar fraction of LPC (Fig. 7C). The contents of PE $O$ - and PG species were decreased as well, whereas small changes in other lipid classed did not reach statistical significance.

Major changes in the patterns of membrane lipid composition between WT and $M f_{s} d 2 a^{-/-}$retinas are summarized in Figure 7, $D$ and $E$. Mfsd2a loss resulted in a $45 \%$ reduction of the DHA content in retinal glycerophospholipids and a $57 \%$ reduction in the total fraction of VLCFAs, with some individual species reduced by over 10-fold (e.g., PC54:12). On the other hand, the retinal content of AA was increased by $66 \%$ and the content of MUFAs by $56 \%$. These changes appear to reflect a compensatory mechanism aiming to maintain a relatively constant overall level of lipid saturation in the retina, perhaps required for maintaining the fluidity of cell membranes.

To confirm that a similar pattern of DHA deficiency takes place specifically in photoreceptor outer segments, we performed lipidomics analysis of rod outer segment membranes isolated from the retinas of both genotypes. Due to a very small amount of available material (particularly from poorly breeding $M f s d 2 a^{+/-}$mice) our quantitative analysis was restricted to four major phospholipid classes (PC, PE, PS, and PI; Fig. 7-1, available at https:// doi.org/10.1523/JNEUROSCI.1142-19. 2019.f7-1). The changes in PC are illustrated in Figure 9A. Overall, we observed an $\sim 56 \%$ loss of phospholipids containing DHA, and compensatory changes, including an $\sim 147 \%$ increase in AA and $\sim 84 \%$ increase in MUFAs (Fig. $9 B$ ). These relative changes are somewhat larger than those observed in whole retinas (for direct comparison, see Fig. 9B), perhaps reflecting the fact that outer segment membranes undergo rapid turnover and are completely replaced in the course of $\sim 10-12 \mathrm{~d}$ in mice (Young, 1967). We also documented a nearly complete loss of major PC species containing two DHA moieties (PC44:12) and VLCFAs containing six double bonds (e.g., PC54:12, PC56:12, PC58:12; Fig. 9A).

This global remodeling of the retinal phospholipid composition could be explained by a combination of altered fatty acid transport, particularly that of DHA, across the blood-retina barrier and an upregulation of desaturase enzymes. The latter was previously documented in the $M f_{s} d 2 a^{-/-}$mouse eyecups analyzed at P13 when retinal development is not yet completed (Wong et al., 2016). To elucidate whether such regulatory mechanisms persist in adult retinas, we used comparative RNA-Seq analysis to evaluate differential gene expression in the retinas of 5- to 6-week-old $M f_{s} d 2 a^{-/-}$mice and their WT littermates (this was the same age at which our lipidome analysis was performed). RNA-Seq data were mapped with DNASTAR, normalized by the RPKM values, and gene expression levels in $M f_{s} d 2 a^{-1-}$ retinas were calculated as percentage of mean values in WT retinas. In agreement with previous report (Wong et al., 2016), we observed an upregulation of Stearoyl-CoA desaturases 1 and 2 (Scd1 and Scd2), fatty acid desaturases 1 and 2 (Fads1 and Fads2), as well as sterol regulatory element-binding factor Srebf1, which reported to control their expression in other experimental systems (Seo et al., 2009; Knebel et al., 2012; Angela et al., 2016; Fig. 10 and Fig. 10-1, available at https://doi.org/10.1523/JNEUROSCI.1142-19. 2019.f10-1). Also in agreement with Wong et al. (2016), we observed a small but statistically significant $\sim 15 \%$ increase in the level of sterol regulatory element-binding factor Srebf2, which controls the expression of genes regulating cholesterol metabolism. A complementary analysis of the RNA-Seq data using the DeSeq2 normalization corroborated statistically significant upregulation of desaturases ( $S c d 1, S c d 2, F a d s 1, F a d s 2)$ and Srebf1, but not Srebf2 (Fig. 10-1, available at https://doi.org/10.1523/ JNEUROSCI.1142-19.2019.f10-1).

Consistent with these findings, the enrichment pathway analysis performed on the data normalized by either RPKM or DeSeq2 (Fig. 10-2, available at https://doi.org/10.1523/JNEUROSCI. 1142-19.2019.f10-2) suggested that $M f_{s} d 2 a^{-/-}$retinas have elevated activity of the pathways, which include lipid desaturases ("Oleate Biosynthesis II" and " $\gamma$-linolenate Biosynthesis II") and various lipid pathways which involve the Srebf1 and/or Srebf2 
transcription factors ("LXR/RXR Activation", "FXR/RXR Activation"). We also noted an upregulation of several genes indicative of ongoing photoreceptor stress (Fig. 10-1, available at https://doi.org/ 10.1523/JNEUROSCI.1142-19.2019.f101), including Stat3 (signal transducer and activator of transcription 3; Jiang et al., 2014), which we use as a representative example in Figure 10.

To control that these changes in the expression of genes regulating lipid metabolism do not merely represent a nonspecific transcriptional response of degenerating retinas, we repeated this analysis for retinas of mice bearing the $\mathrm{P} 23 \mathrm{H}$ mutation in one copy of the rhodopsin gene ( $R h o^{P 23 H / W T}$ mouse), a model commonly used to study retinal degeneration (Sakami et al., 2011). No changes in transcription levels of any of these genes were found, whereas Stat3 was robustly elevated (Fig. 10). This suggests that upregulation of the genes regulating lipid metabolism represents a specific response to the Mfsd2a knock-out.

Importantly, we did not detect any changes in the mRNA level of the Elovl4 gene (the elongation of very long chain fatty acids protein 4) responsible for VLCFA production. This suggests that the loss of phospholipids containing VLCFA is likely caused by a decreased availability of PUFAs, including DHA, serving among fatty acid precursors in the VLCFA biosynthetic pathway (Yu et al., 2012). Similarly, no changes were detected in the mRNA expression level of the Adiporl gene (the Adiponectin Receptor 1), which was suggested to play a role in DHA retention in rods (Rice et al., 2015), and the Acsl6 gene coding Acyl-CoA synthetase 6 recently shown to contribute to DHA enrichment in the brain (Fernandez et al., 2018).

\section{Single-cell recordings from $M f_{s} d 2 a^{-/-}$rods}

In the last set of experiments, we investigated the functional consequences of DHA loss on the sensitivity and recovery kinetics of rod phototransduction, the latter of which is known to be highly dependent on the diffusional encounter of the RGS9 complex in the disc membrane (Krispel et al., 2006; Gospe et al., 2011). The prevalent, although untested in intact cells, hypothesis is that high DHA content provides exceptional photoreceptor membrane liquidity (Treen et al., 1992; Niu et al., 2004), which supports a rapid rate of protein diffusion on these membranes' surface assuring the rapid diffusion needed for high amplification and timely deactivation. $M f_{s} d 2 a^{-/-}$mice provide a unique opportunity to test this hypothesis directly.

Suction electrode recording from intact rods of $M f s d 2 a^{-/-}$ and WT littermate mice revealed light-evoked responses that were highly similar (Fig. 11). Families of responses to flashes that ranged over $3 \log$ units of intensity were qualitatively indistinguishable (Fig. 11A), although on average $M f_{s} d 2 a^{-/-}$rods had smaller maximal dark currents $\left(p=1.1 \times 10^{-8}\right.$; Table 1$)$ and captured a smaller fraction of incident photons ("effective collecting area"; $p=4.7 \times 10^{-3}$; Table 1 ) than their WT counterparts, both of which may have been influenced by the younger average age of the knock-out mice ( 34 vs $50 \mathrm{~d}$; Table $1 ; p=9.3 \times$ $\left.10^{-6}\right)$. Nevertheless, the amplitude and kinetics of the lightevoked responses of $M f_{s} d 2 a^{-/-}$and WT rods were virtually indistinguishable (Table 1), especially when comparing littermates of nearly the exact same ages (Fig. $11 B, C$ ).

\section{Discussion}

In this study, we analyzed the retinal phenotype of a mouse lacking Mfsd2a, an LPC membrane transporter serving as a major contributor to the DHA delivery across both blood-retina and blood-brain barriers (Nguyen et al., 2014; Wong et al., 2016). Perhaps the most striking observation in our study is how relatively normal the functioning of $M f_{s} d 2 a^{-/-}$retinas is, given that they lack $\sim$ one-half of their normal DHA content and that brain pathology in these mice is much more severe (Nguyen et al., 2014; Chan et al., 2018). The progression of photoreceptor loss in $M f_{s} d 2 a^{-1-}$ retinas is relatively slow, particularly compared with many other models of inherited retinal degeneration, and surviving photoreceptors have normal morphology and generate essentially normal light responses.

The retinal phenotype of $M f s d 2 a^{-/-}$mice was previously investigated by (Wong et al., 2016). Whereas our findings are mostly in agreement, we present several unique results and report notable differences that need to be highlighted. Whereas Wong et al. (2016) conducted lipidome analysis using whole eyes, we performed this analysis using isolated retinas and purified outer segments. This allowed us to obtain a more quantitative account of the changes in lipid composition taking place at the retinal side of the blood-retina barrier. Accordingly, we observed an $\sim$ threefold reduction in the overall DHA/AA ratio in $M f s d 2 a^{-/-}$retinas versus an $\sim 1$.9-fold reduction previously measured in whole eyes. Likely for the same reason, we were able to reveal a robust $\sim 56 \%$ 
Table 1. Summary of single rod cell recordings from $M f s d 2 a^{-1-}$ and WT mice

\begin{tabular}{|c|c|c|c|c|c|c|c|}
\hline & $I_{\text {dark }}, \mathrm{pA}$ & $\begin{array}{l}\text { SPR amplitude, } \\
\text { pA }\end{array}$ & $\begin{array}{l}\text { Dim flash } \\
\tau_{\text {rec }}, \mathrm{ms}\end{array}$ & $\begin{array}{l}\text { Dim flash } \\
\text { integration time, ms }\end{array}$ & $I_{0}$, photons $/ \mu \mathrm{m}^{2}$ & $\begin{array}{l}\text { Collecting } \\
\text { area, } \mu \mathrm{m}^{2}\end{array}$ & Age, d \\
\hline WT & $13.8 \pm 0.5(40)$ & $0.91 \pm 0.08(31)$ & $246 \pm 9.7(39)$ & $344 \pm 16.4(40)$ & $35 \pm 1.7(40)$ & $0.45 \pm 0.03(31)$ & $50.8 \pm 2.2(38)$ \\
\hline$M f s d 2 a^{-1-}$ & $9.5 \pm 0.4(45)^{*}$ & $0.76 \pm 0.05(32)$ & $217 \pm 10.9(43)$ & $309 \pm 14.2(44)$ & $38 \pm 2.0(44)$ & $0.33 \pm 0.03(33)^{*}$ & $34.1 \pm 2.7(44)$ \\
\hline
\end{tabular}

Data are shown as mean $\pm S D$ with the number of cells analyzed in parentheses. $I_{\text {dark }}$, Circulating dark current; $S P R$, single-photon response; $\tau_{\text {rec }}$, dim flash time constant of recovery; $I_{0}$, flash strength that elicited a half-maximal response; $\tau_{\mathrm{D}}$, the dominant constant of photoresponse recovery from saturating flashes. ${ }^{*} p \leq 0.05$.

increase in the fraction of phospholipids containing MUFAs. In addition, our lipidomics methodology was suited to quantitatively detect lipids containing VLCFA moieties, which revealed an $\sim 57 \%$ reduction in VLCFAs (not analyzed in the previous study). The corresponding changes in outer segment phospholipids were even more pronounced: an $\sim 5$.6-fold reduction in the DHA/AA ratio and $\sim 84 \%$ MUFA increase. Overall, these changes appear to reflect a compensatory response by the retina aiming to maintain a certain saturation level of the fatty acid moieties after losing a normal route of the DHA supply.

The DHA loss in $M f_{s} d 2 a^{-1-}$ retinas could be sufficiently explained by impaired LPC transport across the blood-retina barrier, and the reduction in VLCFAs could take place for the same reason because DHA serves as one of the VLCFA biosynthesis precursor (Yu et al., 2012). The other major changes in the retinal lipidome are more complex and could be attributed, at least in part, to a transcriptionally-driven attempt by the retina to rebuild its membrane composition. Indeed, the increased proportion of MUFAs could reflect upregulation of the desaturases, SCD1 and SCD2, which catalyze MUFA formation from saturated fatty acids. Consistently, we observed a small $7 \%$ reduction in the saturated fatty acid content, which almost reached statistical significance $(p=0.057)$. The upregulation of AA and complex changes in profiles of fatty acids with 2, 3 and 5 double bonds could be explained by upregulation of desaturases FADS1 and FADS2.

This type of transcriptional response triggered by deficiency of DHA and other PUFAs has been documented in other experimental systems and is thought to restore membrane fluidity and structure upon global or specific lipid deprivation (for review, see Hagen et al., 2010). Of particular interest is a recent biophysical study directly demonstrating the ability of cells to adjust their lipidome in response to PUFA challenge, resulting in the preservation of membrane packing and fluidity (Levental et al., 2018). This response is also consistent with a feedback mechanism in which LPC-DHA suppresses SREBF1 activity in developing brain, whereas Mfsd2a knock-out upregulates SREBF1 and its downstream targets following LPC-DHA deprivation (Chan et al., 2018).

Another unanswered question relates to the mechanisms accounting for the remaining retinal DHA in $M f_{s} d 2 a^{-1-}$ mice. These mechanisms may include developmental DHA accretion before the blood-retina barrier is established with subsequent DHA recycling from the RPE by interphotoreceptor retinoidbinding protein (Chen et al., 1993), or cross-barrier delivery of non-esterified DHA by fatty acid transporters (e.g., CD36 and FATP1; Mitchell and Hatch, 2011; Chouinard-Watkins et al., 2018).

Impaired LPC transport to the retina results in partial photoreceptor loss. However, neither gross morphological abnormalities in $M f s d 2 a^{-/-}$photoreceptors and RPE nor partial rhodopsin mislocalization from rod outer segments of young mice (Wong et al., 2016) were observed in our study. Regardless, both studies documented the invasion of mononuclear phagocytes into the subretinal space, which is a nonspecific hallmark of ongoing photoreceptor pathology (Rathnasamy et al., 2019).

Despite ongoing photoreceptor cell loss, the functional behavior of surviving cells is remarkably normal. This was first suggested by the ERG analysis by Wong et al. (2016). They observed somewhat reduced amplitudes of ERG a- and b-waves but noted that this reduction did not reach statistical significance and could be explained by partial photoreceptor loss. Considering the long history of presumed connection between DHA and normal vision, we were intrigued by this observation and performed singlecell recordings from $M f_{s} d 2 a^{-/-}$rods. Unlike ERG, single-cell recordings are not affected by the fraction of surviving rods and allow precise monitoring of the entire photoresponse time course. The amplitudes and kinetics of light-responses by $M f_{s} d 2 a^{-1-}$ rods are strikingly similar to those from WT rods. This result was unexpected, considering that photoreceptor disc membranes harboring the phototransduction machinery are among the most DHA-enriched in our body (Fliesler and Anderson, 1983). Yet, such an unusually high DHA content does not appear to directly serve the needs of phototransduction and may relate to another enigmatic aspect of these cells' function not immediately related to light detection.

So, what causes degeneration of $M f s d 2 a^{-/-}$rods? While obtaining a definitive answer is another subject of future investigation, we can propose several not mutually exclusive mechanisms. A rather trivial explanation is that these cells suffer from a general lipid insufficiency caused by impaired LPC transport. Such insufficiency would particularly affect photoreceptors since they replenish the entire complement of outer segment membranes every 10-12 d (Young, 1967). Consistent with this explanation, the knock-outs of LPCAT1 or LPAAT3, the enzymes regulating the biosynthesis of retinal phospholipids (including but not limited to those containing DHA), cause even more severe photoreceptor degeneration than the Mfsd2a knock-out (Friedman et al., 2010; Shindou et al., 2017). Another potential explanation is that reduced lipid supply to the $M f_{s} d 2 a^{-/-}$retina causes energy deprivation since the retina, and particularly photoreceptors, can oxidize lipid through fatty acid $\beta$-oxidation to sustain its energy needs (Joyal et al., 2016). It is also possible that pathology arises from significant changes in fatty acids (MUFAs, DHA, AA and VLCFAs), which act as signaling molecules, either themselves or through their metabolites (Igal, 2016; Rodríguez-Cruz and Serna, 2017; Bazan, 2018). For example, the shift in the DHA/AA ratio may be proinflammatory (SanGiovanni and Chew, 2005). Of particular interest is the observed reduction in VLCFAs, as evolving evidence suggests a complex involvement of VLCFAs in supporting retinal structure and function, particularly in the context of hereditary retinal degenerations, including Stargardt-3 macular degeneration (Hopiavuori et al., 2019). Finally, a recent study reported that Mfsd2a suppresses transcellular transport in endothelial cells of the retinal vasculature (Chow and $\mathrm{Gu}, 2017$ ). One can imagine that this may be detrimental for the retina, although it is not clear how exactly increased transcytosis in retinal vasculature may lead to photoreceptor degeneration. 
Another important conclusion from our study is that the consequences of DHA deficit caused by the Mfsd2a knock-out are different from those caused by dietary DHA restriction. Dietary restriction in mice and rats could lead to a higher degree of DHA deprivation of the retina associated with reductions in ERG responses, outer segment shortening and alteration in the rate of photoreceptor outer segment renewal without notable photoreceptor loss (Benolken et al., 1973; Wheeler et al., 1975; Senapati et al., 2018). However, this diet leads to a major substitution of DHA with docosapentaenoic acid (22:5; Anderson et al., 1974; Niu et al., 2004), which does not happen in $M f_{s} d 2 a^{-/-}$retinas. Therefore, any direct comparison of retinal DHA deprivation through dietary restriction versus Mfsd2a knock-out may be deceiving.

In a similar context, the study by Rice et al. (2015) analyzed the retinal phenotype of the adiponectin receptor 1 (Adipor1) knockout and found an $\sim 50 \%$ reduction in the DHA content associated with a more severe photoreceptor degeneration than in Mfsd2a knock-out. The authors concluded that the activity of ADIPOR1 is critical for DHA retention in the retina and RPE.

In summary, our results, combined with other observations discussed here, argue that maintaining an efficient phospholipid supply to the retina is more critical for supporting photoreceptor survival than their ability to produce light responses. Furthermore, the degree of photoreceptor pathology does not appear to directly correlate with the degree of retinal DHA deprivation and may be driven by other concurrent changes in the retinal lipidome. Therefore, the challenges of future studies are to pinpoint the precise place of DHA, other unsaturated fatty acids and their metabolites in the complex regulatory networks defining the compositions of retinal and photoreceptor lipidomes and to identify potential targets within these networks for improving photoreceptor health in congenital and age-related diseases of the retina.

\section{References}

Alakbarzade V, Hameed A, Quek DQ, Chioza BA, Baple EL, CazenaveGassiot A, Nguyen LN, Wenk MR, Ahmad AQ, Sreekantan-Nair A, Weedon MN, Rich P, Patton MA, Warner TT, Silver DL, Crosby AH (2015) A partially inactivating mutation in the sodium-dependent lysophosphatidylcholine transporter MFSD2A causes a non-lethal microcephaly syndrome. Nat Genet 47:814-817.

Anderson RE, Benolken RM, Dudley PA, Landis DJ, Wheeler TG (1974) Proceedings: polyunsaturated fatty acids of photoreceptor membranes. Exp Eye Res 18:205-213.

Angela M, Endo Y, Asou HK, Yamamoto T, Tumes DJ, Tokuyama H, Yokote K, Nakayama T (2016) Fatty acid metabolic reprogramming via mTORmediated inductions of PPAR $\gamma$ directs early activation of T cells. Nat Commun 7:13683.

Bazan NG (2018) Docosanoids and elovanoids from omega-3 fatty acids are pro-homeostatic modulators of inflammatory responses, cell damage and neuroprotection. Mol Aspects Med 64:18-33.

Bazan NG, Molina MF, Gordon WC (2011) Docosahexaenoic acid signalolipidomics in nutrition: significance in aging, neuroinflammation, macular degeneration, Alzheimer's, and other neurodegenerative diseases. Annu Rev Nutr 31:321-351.

Benedicto I, Lehmann GL, Ginsberg M, Nolan DJ, Bareja R, Elemento O, Salfati Z, Alam NM, Prusky GT, Llanos P, Rabbany SY, Maminishkis A, Miller SS, Rafii S, Rodriguez-Boulan E (2017) Concerted regulation of retinal pigment epithelium basement membrane and barrier function by angiocrine factors. Nat Commun 8:15374.

Benolken RM, Anderson RE, Wheeler TG (1973) Membrane fatty acids associated with the electrical response in visual excitation. Science 182: 1253-1254.

Ben-Zvi A, Lacoste B, Kur E, Andreone BJ, Mayshar Y, Yan H, Gu C (2014) $\mathrm{Mfsd} 2 \mathrm{a}$ is critical for the formation and function of the blood-brain barrier. Nature 509:507-511.
Chan JP, Wong BH, Chin CF, Galam DLA, Foo JC, Wong LC, Ghosh S, Wenk MR, Cazenave-Gassiot A, Silver DL (2018) The lysolipid transporter Mfsd2a regulates lipogenesis in the developing brain. PLoS Biol 16:e2006443

Chen Y, Saari JC, Noy N (1993) Interactions of all-trans-retinol and longchain fatty acids with interphotoreceptor retinoid-binding protein. Biochemistry 32:11311-11318.

Chouinard-Watkins R, Lacombe RJS, Bazinet RP (2018) Mechanisms regulating brain docosahexaenoic acid uptake: what is the recent evidence? Curr Opin Clin Nutr Metab Care 21:71-77.

Chow BW, Gu C (2017) Gradual suppression of transcytosis governs functional blood-retinal barrier formation. Neuron 93:1325-1333.e3.

Fernandez RF, Kim SQ, Zhao Y, Foguth RM, Weera MM, Counihan JL, Nomura DK, Chester JA, Cannon JR, Ellis JM (2018) Acyl-CoA synthetase 6 enriches the neuroprotective omega- 3 fatty acid DHA in the brain. Proc Natl Acad Sci U S A 115:12525-12530.

Fliesler SJ, Anderson RE (1983) Chemistry and metabolism of lipids in the vertebrate retina. Prog Lipid Res 22:79-131.

Friedman JS, Chang B, Krauth DS, Lopez I, Waseem NH, Hurd RE, Feathers KL, Branham KE, Shaw M, Thomas GE, Brooks MJ, Liu C, Bakeri HA, Campos MM, Maubaret C, Webster AR, Rodriguez IR, Thompson DA, Bhattacharya SS, Koenekoop RK, et al. (2010) Loss of lysophosphatidylcholine acyltransferase 1 leads to photoreceptor degeneration in $\mathrm{rd} 11$ mice. Proc Natl Acad Sci U S A 107:15523-15528.

Gospe SM 3rd, Baker SA, Kessler C, Brucato MF, Winter JR, Burns ME, Arshavsky VY (2011) Membrane attachment is key to protecting transducin GTPase-activating complex from intracellular proteolysis in photoreceptors. J Neurosci 31:14660-14668.

Gross OP, Burns ME (2010) Control of rhodopsin's active lifetime by arrestin-1 expression in mammalian rods. J Neurosci 30:3450-3457.

Guemez-Gamboa A,Nguyen LN, Yang H, Zaki MS, Kara M, Ben-Omran T, Akizu N, Rosti RO, Rosti B, Scott E, Schroth J, Copeland B, Vaux KK, Cazenave-Gassiot A, Quek DQ, Wong BH, Tan BC, Wenk MR, Gunel M, Gabriel S, et al. (2015) Inactivating mutations in MFSD2A, required for omega-3 fatty acid transport in brain, cause a lethal microcephaly syndrome. Nat Genet 47:809-813.

Hagen RM, Rodriguez-Cuenca S, Vidal-Puig A (2010) An allostatic control of membrane lipid composition by SREBP1. FEBS Lett 584:2689-2698.

Harel T, Quek DQY, Wong BH, Cazenave-Gassiot A, Wenk MR, Fan H, Berger I, Shmueli D, Shaag A, Silver DL, Elpeleg O, Edvardson S (2018) Homozygous mutation in MFSD2A, encoding a lysolipid transporter for docosahexanoic acid, is associated with microcephaly and hypomyelination. Neurogenetics 19:227-235.

Herzog R, Schwudke D, Schuhmann K, Sampaio JL, Bornstein SR, Schroeder M, Shevchenko A (2011) A novel informatics concept for highthroughput shotgun lipidomics based on the molecular fragmentation query language. Genome Biol 12:R8.

Hopiavuori BR, Anderson RE, Agbaga MP (2019) ELOVL4: very long-chain fatty acids serve an eclectic role in mammalian health and function. Prog Retin Eye Res 69:137-158.

Igal RA (2016) Stearoyl CoA desaturase-1: new insights into a central regulator of cancer metabolism. Biochim Biophys Acta 1861:1865-1880.

Jiang K, Wright KL, Zhu P, Szego MJ, Bramall AN, Hauswirth WW, Li Q, Egan SE, McInnes RR (2014) STAT3 promotes survival of mutant photoreceptors in inherited photoreceptor degeneration models. Proc Natl Acad Sci U S A 111:E5716-E5723.

Joly S, Lange C, Thiersch M, Samardzija M, Grimm C (2008) Leukemia inhibitory factor extends the lifespan of injured photoreceptors in vivo. J Neurosci 28:13765-13774.

Joyal JS,Sun Y, Gantner ML, Shao Z, Evans LP, Saba N, Fredrick T, Burnim S, Kim JS, Patel G, Juan AM, Hurst CG, Hatton CJ, Cui Z, Pierce KA, Bherer P, Aguilar E, Powner MB, Vevis K, Boisvert M, et al. (2016) Retinal lipid and glucose metabolism dictates angiogenesis through the lipid sensor Ffar1. Nat Med 22:439-445.

Knebel B, Haas J, Hartwig S, Jacob S, Köllmer C, Nitzgen U, Muller-Wieland D, Kotzka J (2012) Liver-specific expression of transcriptionally active SREBP-1c is associated with fatty liver and increased visceral fat mass. PLoS One 7:e31812.

Krispel CM, Chen D, Melling N, Chen YJ, Martemyanov KA, Quillinan N, Arshavsky VY, Wensel TG, Chen CK, Burns ME (2006) RGS expression rate-limits recovery of rod photoresponses. Neuron 51:409-416.

Levental KR, Malmberg E, Ernst R, Levental I (2018) Homeostatic remod- 
eling of mammalian membranes in response to dietary lipids is essential for cellular fitness. BioRxiv. Available at http://www.biorxiv.org/content/ 10.1101/342873v1.full.

Lobanova ES, Finkelstein S, Skiba NP, Arshavsky VY (2013) Proteasome overload is a common stress factor in multiple forms of inherited retinal degeneration. Proc Natl Acad Sci U S A 110:9986-9991.

Lobanova ES, Finkelstein S, Li J, Travis AM, Hao Y, Klingeborn M, Skiba NP, Deshaies RJ, Arshavsky VY (2018) Increased proteasomal activity supports photoreceptor survival in inherited retinal degeneration. Nat Commun 9:1738.

Mitchell RW, Hatch GM (2011) Fatty acid transport into the brain: of fatty acid fables and lipid tails. Prostaglandins Leukot Essent Fatty Acids 85:293-302.

Mudhar HS, Pollock RA, Wang C, Stiles CD, Richardson WD (1993) PDGF and its receptors in the developing rodent retina and optic nerve. Development 118:539-552.

Nguyen LN, Ma D, Shui G, Wong P, Cazenave-Gassiot A, Zhang X, Wenk MR, Goh EL, Silver DL (2014) Mfsd2a is a transporter for the essential omega-3 fatty acid docosahexaenoic acid. Nature 509:503-506.

Niu SL, Mitchell DC, Lim SY, Wen ZM, Kim HY, Salem N Jr, Litman BJ (2004) Reduced G protein-coupled signaling efficiency in retinal rod outer segments in response to n-3 fatty acid deficiency. J Biol Chem 279:31098-31104.

Petters RM, Alexander CA, Wells KD, Collins EB, Sommer JR, Blanton MR, Rojas G, Hao Y, Flowers WL, Banin E, Cideciyan AV, Jacobson SG, Wong F (1997) Genetically engineered large animal model for studying cone photoreceptor survival and degeneration in retinitis pigmentosa. Nat Biotechnol 15:965-970.

Rathnasamy G, Foulds WS, Ling EA, Kaur C (2019) Retinal microglia: a key player in healthy and diseased retina. Prog Neurobiol 173:18-40.

Rice DS, Calandria JM, Gordon WC, Jun B, Zhou Y, Gelfman CM, Li S, Jin M, Knott EJ, Chang B, Abuin A, Issa T, Potter D, Platt KA, Bazan NG (2015) Adiponectin receptor 1 conserves docosahexaenoic acid and promotes photoreceptor cell survival. Nat Commun 6:6228.

Rodríguez-Cruz M, Serna DS (2017) Nutrigenomics of omega-3 fatty acids: regulators of the master transcription factors. Nutrition 41:90-96.

Sakami S, Maeda T, Bereta G, Okano K, Golczak M, Sumaroka A, Roman AJ, Cideciyan AV, Jacobson SG, Palczewski K (2011) Probing mechanisms of photoreceptor degeneration in a new mouse model of the common form of autosomal dominant retinitis pigmentosa due to $\mathrm{P} 23 \mathrm{H}$ opsin mutations. J Biol Chem 286:10551-10567.

Sales S, Graessler J, Ciucci S, Al-Atrib R, Vihervaara T, Schuhmann K, Kauhanen D, Sysi-Aho M, Bornstein SR, Bickle M, Cannistraci CV, Ekroos K, Shevchenko A (2016) Gender, contraceptives and individual metabolic predisposition shape a healthy plasma lipidome. Sci Rep 6:27710.

SanGiovanni JP, Chew EY (2005) The role of omega-3 long-chain polyunsaturated fatty acids in health and disease of the retina. Prog Retin Eye Res 24:87-138.

Sato T (1968) A modified method for lead staining of thin sections. J Electron Microsc 17:158-159.

Schuhmann K, Herzog R, Schwudke D, Metelmann-Strupat W, Bornstein SR,
Shevchenko A (2011) Bottom-up shotgun lipidomics by higher energy collisional dissociation on LTQ orbitrap mass spectrometers. Anal Chem 83:5480-5487.

Schuhmann K, Almeida R, Baumert M, Herzog R, Bornstein SR, Shevchenko A (2012) Shotgun lipidomics on a LTQ orbitrap mass spectrometer by successive switching between acquisition polarity modes. J Mass Spectrom 47:96-104.

Schuhmann K, Thomas H, Ackerman JM, Nagornov KO, Tsybin YO, Shevchenko A (2017) Intensity-independent noise filtering in FT MS and FT MS/MS spectra for shotgun lipidomics. Anal Chem 89:70467052.

Scott BL, Bazan NG (1989) Membrane docosahexaenoate is supplied to the developing brain and retina by the liver. Proc Natl Acad Sci U S A 86:2903-2907.

Senapati S, Gragg M, Samuels IS, Parmar VM, Maeda A, Park PS (2018) Effect of dietary docosahexaenoic acid on rhodopsin content and packing in photoreceptor cell membranes. Biochim Biophys Acta Biomembr 1860:1403-1413.

Seo YK, Chong HK, Infante AM, Im SS, Xie X, Osborne TF (2009) Genomewide analysis of SREBP-1 binding in mouse liver chromatin reveals a preference for promoter proximal binding to a new motif. Proc Natl Acad Sci U S A 106:13765-13769.

Shindou H, Koso H, Sasaki J, Nakanishi H, Sagara H, Nakagawa KM, Takahashi Y, Hishikawa D, Iizuka-Hishikawa Y, Tokumasu F, Noguchi H, Watanabe S, Sasaki T, Shimizu T (2017) Docosahexaenoic acid preserves visual function by maintaining correct disc morphology in retinal photoreceptor cells. J Biol Chem 292:12054-12064.

Treen M, Uauy RD, Jameson DM, Thomas VL, Hoffman DR (1992) Effect of docosahexaenoic acid on membrane fluidity and function in intact cultured Y-79 retinoblastoma cells. Arch Biochem Biophys 294:564-570.

Tsang SH, Burns ME, Calvert PD, Gouras P, Baylor DA, Goff SP, Arshavsky VY (1998) Role for the target enzyme in deactivation of photoreceptor G protein in vivo. Science 282:117-121.

Vanlandewijck M, He L, Mäe MA, Andrae J, Ando K, Del Gaudio F, Nahar K, Lebouvier T, Laviña B, Gouveia L, Sun Y, Raschperger E, Räsänen M, Zarb Y, Mochizuki N, Keller A, Lendahl U, Betsholtz C (2018) A molecular atlas of cell types and zonation in the brain vasculature. Nature 554:475-480.

Wheeler TG, Benolken RM, Anderson RE (1975) Visual membranes: specificity of fatty acid precursors for the electrical response to illumination. Science 188:1312-1314.

Wong BH, Chan JP, Cazenave-Gassiot A, Poh RW, Foo JC, Galam DL, Ghosh S, Nguyen LN, Barathi VA, Yeo SW, Luu CD, Wenk MR, Silver DL (2016) Mfsd2a is a transporter for the essential omega-3 fatty acid docosahexaenoic acid (DHA) in eye and is important for photoreceptor cell development. J Biol Chem 291:10501-10514.

Young RW (1967) The renewal of photoreceptor cell outer segments. J Cell Biol 33:61-72.

Yu M, Benham A, Logan S, Brush RS, Mandal MN, Anderson RE, Agbaga MP (2012) ELOVL4 protein preferentially elongates $20: 5 \mathrm{n} 3$ to very long chain PUFAs over 20:4n6 and 22:6n3. J Lipid Res 53:494-504. 